\title{
Aerosol nucleation in a turbulent jet using Large Eddy Simulations
}

\author{
I. Pesmazoglou \\ Department of Mechanical Engineering, Imperial College, London SW7 $2 A Z$ \\ A. M. Kempf \\ Chair of fluid dynamics, Institute for Combustion and Gasdynamics, and Center for \\ Computational Sciences and Simulation, Universität Duisburg-Essen, Duisburg, 47048 \\ S. Navarro-Martinez* \\ Department of Mechanical Engineering, Imperial College, London SW7 $2 A Z$
}

\begin{abstract}
This work presents a model for particle nucleation and transport in the context of Large Eddy Simulation. A turbulent Dibutyl-Phthalate-laden Nitrogen jet diffusing in atmospheric air is used for validation. The proposed nucleation model treats the process in a probabilistic manner where the frequency of events is determined from local equilibrium conditions. Two methodologies for the sub-grid influence on nucleation rates are implemented: the presumed $\beta$-PDF and the source expansion method. Good agreement is found with respect to the experimental results for particle concentrations. The differences between using mean and instantaneous values used for the evaluation of the nucleation rate are shown. For the grid spacing used, the unresolved scales seem to have little influence on the calculated particle concentrations. It is
\end{abstract}

\footnotetext{
*Corresponding author:

Email address: s.navarro@imperial.ac.uk (S. Navarro-Martinez )
} 
concluded that the use of instantaneous nucleation rates is advantageous and therefore it is important to consider a particle coupling that allows for the full use of instantaneous values.

Keywords: LES, Nucleation, Turbulent Jet, Particles, Lagrangian, Aerosol

\section{Introduction}

Uniform phase processes may reach a supersaturated state which suddenly collapses due to a phase transition of the supersaturated vapour to an aerosol. This process is termed nucleation and it involves the use of either a nucleus composed of vapour molecules of the same material, or an exogenous (to the vapour) surface such as an ionic cluster, an impurity on the surrounding enclosure (e.g. bubbles formed at the surface of a glass of sparkling water), or a small particle of a different material (e.g. dust particles).

Nucleation is the driving process in a number of industrial and atmospheric applications, such as soot formation in combustion processes and fugitive emissions from industrial sources [1], nano-particle synthesis $[2,3]$, and crystal precipitation in liquid phase reactors, among others. All these

processes highly depend on nucleation rates [4]. A correct description of nucleation and growth processes may lead to a successful retrieval of the Particle Size Distribution (PSD). The ability to influence or control the PSD is a very useful tool for generating filter test particles [5] or for preventing health-related effects of newly formed particles [6].

Homogeneous nucleation describes the process whereby one or more gases condense without the aid of an exogenous surface to form particles. For homogeneous nucleation to occur, the vapour must first reach a supersaturated 
state. Thereafter, a small 'embryo' must form that will allow additional vapour molecules to attach and cause the embryo to grow. Nucleation embryos are continuously formed and disintegrated by random processes; depending on the local thermodynamic conditions, a stable nucleus might form and grow to become a particle [7].

Any first order phase transition requires the surmounting of an energy barrier. A fluctuation in temperature, pressure, or concentration modifies the activation energy and therefore locally modifies the rate of phase transition. This process makes nucleation rates extremely sensitive to variations in local thermodynamic properties [8]. This non-linearity is the primary challenge and incentive for the examination of nucleation events and there is an increasing effort in exploring the phenomenon.

Direct measurement of nucleation rates is difficult in non-stationary environments. Although there are a number of ways to retrieve the PSD and concentration densities (e.g. with the use of a Condensation Particle Counter), the rate of particle formation at an exact location cannot be measured directly. This occurs because at any position of the domain, the measured instantaneous concentration includes particles that nucleated upstream and were subsequently transported to that location. A deeper understanding of the nucleation process therefore requires a close examination of experimental data along with rigorous computational modelling. Early investigations of turbulent-coupled nucleation were almost exclusively theoretical or experimental. A description of species concentration, temperature, and velocity fluctuations was limited; nucleation rates were therefore rendered unreliable [9]. With the recent advance in computational power various approaches have 
been introduced for the coupling of the particulate phase evolution with a turbulent flow.

For the investigation of a turbulent-flow-coupled nucleation rate, In the present work, a supersaturated Dibutyl-Phthalate (DBP)-laden Nitrogen $\left(\mathrm{N}_{2}\right)$ turbulent jet is investigated [10]. DBP is a stable compound with low toxicity, and it can be considered representative of a heavy organic fugitive emission. DBP has been favored in nucleation studies, $[11,12,13,14,15,16]$ among others, because it can reach high supersaturations with low concentrations. The turbulent jet studied is a configuration with many practical applications in industry, ranging from prototype small-scale injectors to industrial chimneys. Furthermore there is a significant amount of literature for its particular flow characteristics, as well as self-similar solutions for the velocities and passive scalar distributions $[17,18,19]$. The experimental test case of Lesniewski and Friedlander [10, 20, 21] was selected. This particular case has been examined in a number of computational studies [4, 22, 9], allowing for further comparison of data and implementation methods.

The aims of this work are: firstly, to propose an implementation of the nucleation theory in the context of a Large Eddy Simulation (LES); and secondly, to investigate the significance of the sub-grid scale contributions on homogeneous nucleation rates. The main advantages of LES are its abilities to provide instantaneous values of the field and to directly resolve the most energetic eddies without a closure model. Retrieving instantaneous data can be very important due to the non-linearity of the nucleation rate.

The following sections summarize the experimental set up and physical characteristics of the test case. Thereafter, the methodology is presented for 
the particle transport and for the various treatments of homogeneous nucleation. The coupling of a non-equilibrium flow to the nucleation rate is investigated and the present method is compared to approaches from relevant literature. Having established the numerical treatment, the results and conclusions are presented.

\section{Experimental Configuration}

Lesniewski and Friedlander [10, 20, 21] used a turbulent diffusion chamber to conduct a series of experiments and measure particle concentrations and size distributions for a DBP-laden Nitrogen jet diffusing in atmospheric air. The Nitrogen stream was heated and passed through a bubbler where it was saturated with DBP at a saturation temperature assumed to be equal to the temperature of the bubbler. The DBP-laden gas was then passed through a filter to remove particles from the carrier phase and sent with high velocity into a chamber via an insulated nozzle with an inner diameter $D_{j e t}$ and outer diameter $D_{\text {ins }}$. An air co-flow entered concentrically into the chamber. An outline of the diffusion chamber is shown in Fig. 1.

The jet was turbulent and subsonic, with velocities varying from 30 to $80 \mathrm{~m} / \mathrm{s}$. In this work a jet bulk velocity of $U_{\text {jet }}=51.5 \mathrm{~m} / \mathrm{s}$ was chosen with a co-flow velocity of $U_{c f}=0.18 \mathrm{~m} / \mathrm{s}$. The pressure was held constant at approximately atmospheric conditions. The jet inlet temperature was measured experimentally using a thermocouple under the nozzle exit and was held at $413 \mathrm{~K}$, while the co-flow air temperature was $299 \mathrm{~K}$.

For a nozzle volumetric flow-rate of $Q_{j e t}=10 \mathrm{lpm}$ the jet engulfed the domain at $Z_{d} / D_{\text {jet }} \approx 90$, where $Z_{d}$ is the axial distance. The nozzle diameter 


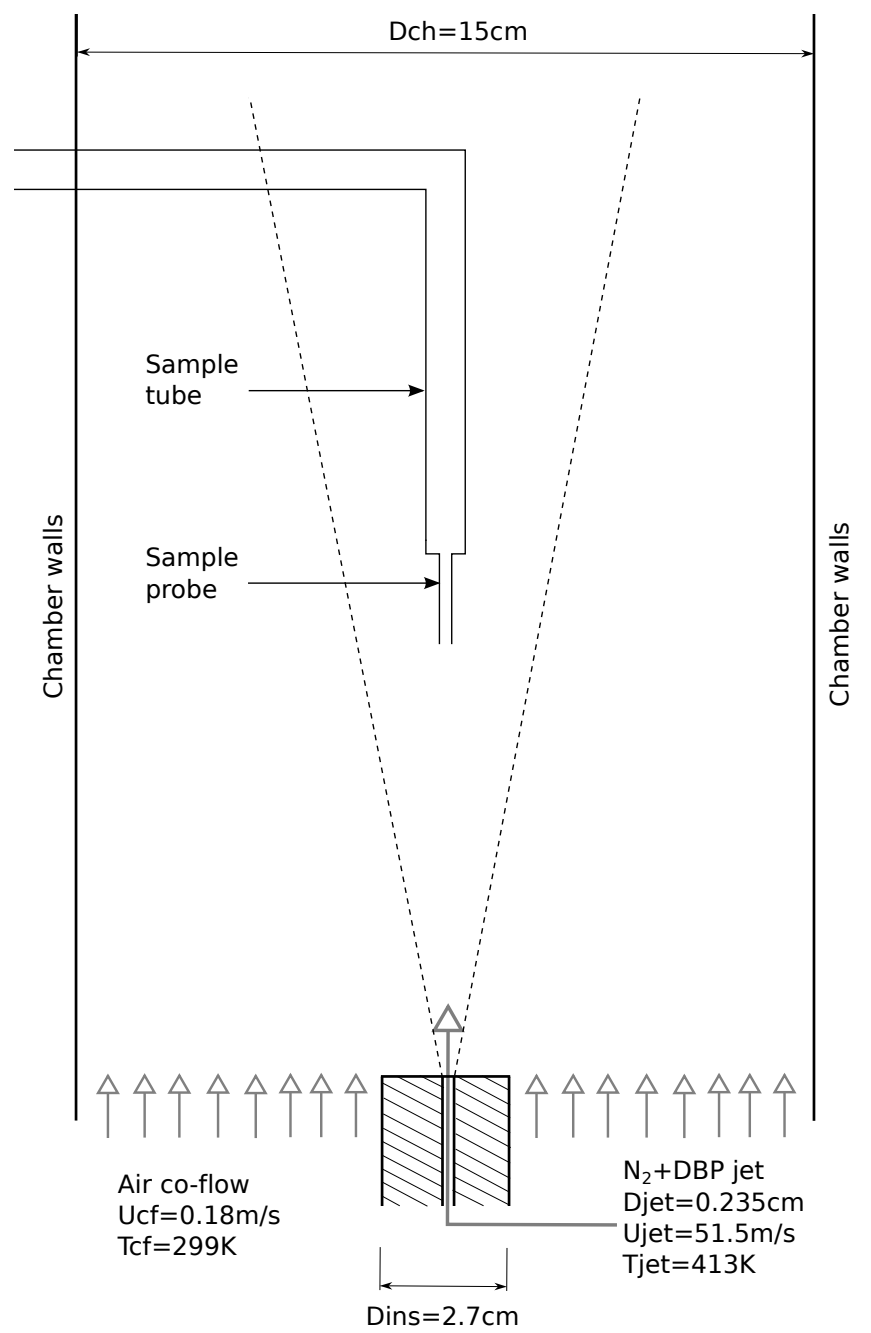

Figure 1: Experimental configuration of [10]. The heated $N_{2}$ DBP-laden jet enters through an insulated nozzle in the diffusion chamber. A concentric flow of air at atmospheric conditions is added. Nucleated particles are sampled and counted using a probe positioned at a number of axial locations leading though a tube to the Condensation Particle Counter $(\mathrm{CPC})$. 
in the experiment was varied. In the context of this work a nozzle diameter of $D_{j e t}=2.35 \mathrm{~mm}$ and an insulator diameter $D_{\text {ins }}=27 \mathrm{~mm}$ were chosen; see Fig. 1. No recirculation was observed near the nozzle outlet in [10]. The experimental chamber had a diameter of $150 \mathrm{~mm}$ and $460 \mathrm{~mm}$ length. The simulated domain had dimensions $50 \times 50 \times 100 \mathrm{~mm}$, with a second simulation performed in a domain of $50 \times 50 \times 200 \mathrm{~mm}$ to compare the particle concentration axial trend with the experimental data.

Measurements of the particle concentrations were taken using a sampling probe positioned in various axial locations. According to Lesniewski and Friedlander [10], the probe did not affect the upstream flow conditions. Once a sample was extracted, it was passed via a stainless steel tube to a Condensation Particle Counter (CPC). The residence time in the tube was $0.3 \mathrm{~s}$; the sample was diluted to prevent further growth of the particles and heated to quench further nucleation. Measurements were taken after the CPC had a steady output for $30 \mathrm{~s}$. The initial DBP mole fraction was measured using a flame ionization detector (FID) and was varied from $1-5 \times 10^{-4}$ in different experimental runs. In this study an initial mole fraction of $3.8 \times 10^{-4}$ was used. Both the FID and CPC data had a $\pm 10 \%$ random error [10].

\section{Methodology}

\subsection{Continuous Phase}

The simulation treats the continuous phase in an Eulerian LES using the in-house incompressible PsiPhi code [23, 24]. For the purpose of the simulation a Lewis number $L e=1$ was assumed. It must be noted that for DBP-laden Nitrogen $L e \approx 5[25]$ and therefore fluid is cooled faster 
than it is diluted, creating a difference in nucleation rates at in laminar regions. At high Reynolds numbers, molecular effects of temperature on fluid properties can be considered negligible when compared to turbulent-driven diffusion, although high Lewis number effects on nucleation may exist. The assumption of unity Lewis number in the present configuration allows for a direct comparison with the methodologies used in similar computational studies $[4,22,9]$.

The condensed vapour was only a small fraction of the total concentration [10], therefore DBP depletion due to nucleation was considered negligible. The unknown sub grid-scale stresses of the momentum equation are closed using the Smagorinsky eddy-viscosity model [26] with the model constant of $C_{s}=0.173$ suggested by Lilly [27]. A Central Differencing Scheme secondorder accurate in space is used for the convective momentum fluxes, a Total Variation Diminishing scheme (TVD) for the convective scalar fluxes, and a third-order Runge-Kutta Scheme is used for integration in time. The timestep width is set according to the Courant-Friedrichs-Lewy (CFL) condition [28], with a constant of $C F L=0.3$. The code uses implicit filtering with equally sized cubic cells, thus ensuring good numerical accuracy and efficient parallelization. A grid-spacing of $\Delta=0.25 \mathrm{~mm}$ is found to sufficiently resolve the jet evolution, which corresponds to roughly 10 points across the jet diameter. Pseudo-turbulence, with a prescribed rms velocity of $10 \%$ the jet bulk velocity and an integral length-scales of half the jet diameter is created by Klein's inflow generator $[29,30]$. The computational time is approximately 15000 CPU hours per simulation. 


\subsection{Particle Transport}

All nucleated particles were assumed spherical with a diameter equivalent to the critical nucleation diameter $d_{p}^{*}$. Itô's equivalent Stochastic Differential Equation (SDE) for stochastic particles is solved following the proposed solution of Jones et al. [31, 32, 33] and implemented in previous work [34] . The particle motion is governed by the Lagrangian equations (1) and (2): the

position $\mathbf{x}_{p}$ of the $p^{\text {th }}$ particle is obtained by the integration of (1) in time, while the incremental change of velocity $\mathbf{v}_{p}$ of the particle is obtained by (2). The ensemble average of all particle paths gives the Eulerian equivalent of the positions and velocities of the particles.

$$
\begin{aligned}
d \mathbf{x}_{p} & =\mathbf{v}_{p} d t \\
d \mathbf{v}_{p} & =\frac{\left(\tilde{\mathbf{U}}_{f}-\mathbf{v}_{\mathbf{p}}\right)}{\tau_{p}} d t+\sqrt{C_{o} \frac{k_{S G S}}{\tau_{t}}} d \mathbf{W}
\end{aligned}
$$

Here, $\tilde{\mathbf{U}}_{f}$ is the LES gas velocity; $C_{o}=1$ is a dispersion constant $[31,32,34]$; the sub grid scale (SGS) kinetic energy $k_{S G S}$ of the gas phase is calculated as $k_{S G S}=2 \Delta^{2} C_{s}^{2 / 3} \tilde{S}_{i j} \tilde{S}_{i j}$ [35], with $\tilde{S}_{i j}$ being the filtered strain tensor; $\tau_{t}$ is a time-scale representative of the disperse and continuous phase turbulent interaction; $\tau_{p}$ is the particle relaxation time; and $d W=\chi \sqrt{d t}$ is the incremental Wiener term, where $\chi$ is a random variable sampled from a normal distribution with mean 0 and variance 1 . The particle relaxation time is determined from:

$$
\tau_{p}^{-1}=\frac{3}{4} \frac{\rho_{f} C_{D}}{\rho_{p} d_{p}}\left|\tilde{\mathbf{U}}_{f}-\mathbf{v}_{\mathbf{p}}\right|
$$

where $\rho_{p}, \rho_{f}$ are the particle and continuous phase densities, respectively, and $d_{p}$ is the particle diameter. The turbulence interaction time-scale is found 
from:

$$
\tau_{t}=\tau_{p}\left(\frac{\tau_{p} k_{S G S}^{1 / 2}}{\Delta}\right)^{2 \alpha-1}
$$

where the constant $\alpha=0.8$, as proposed in [35, 36, 37].

The drag coefficient, $C_{D}$, is defined by the drag law of Yuen-Chen [38], assuming spherical particles and using an empirical relation based on the particle Reynolds number $R e_{p}=\rho_{p} d_{p}\left|\tilde{\mathbf{U}}_{f}-\mathbf{v}_{\mathbf{p}}\right| / \mu$, viz:

$$
C_{D}= \begin{cases}\frac{24}{R e_{p}}\left(1+\frac{R e_{p}^{2 / 3}}{6}\right) & R e_{p}<1000 \\ 0.424 & R e_{p}>1000\end{cases}
$$

The Brownian diffusivity of nucleated particles is estimated to be $\mathcal{D}_{p} \sim$ $\mathcal{O}\left(10^{-6}\right)$ based on the jet temperature and $d_{p}=2 \mathrm{~nm}$, (with a Cunningham slip correction of 100). This diffusivity can be considered negligible compared to the eddy diffusivity, $\mathcal{D}_{\text {eddy }} \sim \mathcal{O}\left(10^{-2}\right)$, and the sub-grid diffusivity represented by Eqn. (2). It has to be noted that for particles in the nano-scale, the relaxation time is very small and the stochastic term is also expected to be small.

For the solution of a given set of particle equations (1-4), the continuous phase properties at the position of a given particle are provided from the Eulerian LES using a linear interpolation of the cell-centred values in the eight neighboring cells.

\subsection{Nucleation Rate}

In the context of this work the Classical Nucleation Theory (CNT) [39, $40,41]$ is used to model nucleation. The nucleation rate $\dot{I}\left[\# / \mathrm{m}^{3} \mathrm{~s}\right]$ is given 
by the CNT as:

$$
\dot{I}=\frac{P_{v} X_{v}}{k_{B} T} \sqrt{\frac{2 \sigma}{\pi m}} \exp \left[\frac{-16 \pi \sigma^{3} m^{2}}{3\left(k_{B} T\right)^{3} \rho_{l}^{2}(\ln S)^{2}}\right] .
$$

The partial pressure and concentration of the nucleating vapor are $P_{v}$ and $X_{v}$, respectively; $S=P_{v} / P_{\text {sat }}$ is the saturation ratio, with the saturation pressure $P_{\text {sat }}$; the surface tension $\sigma$ and condensed density $\rho_{l}$ are retrieved from experimentally fitted functions depending on the nucleating species; $T$ is the temperature in Kelvin; $k_{B}$ is the Boltzmann constant; and $m$ is the mass of a monomer, given by $m=M / N_{\text {avg }}$, where $M$ is the molecular weight of the condensing species and $N_{\text {avg }}$ is Avogadro's number.

Equation (6) stems from a thermodynamic equilibrium model, viz:

$$
\dot{I}=I_{o} \exp \left(-\Delta G_{h o m} / k_{B} T\right)
$$

where: $I_{o}$ is a non-exponential scaling function; and $\Delta G_{h o m}$ is the thermodynamic barrier to homogeneous nucleation and represents the increase of free energy of a system due to the formation of a nucleus of critical size $d_{p}^{*}$, which is given by:

$$
d_{p}^{*}=\frac{4 \sigma v_{m}}{k_{B} T \ln S},
$$

where $v_{m}\left[\mathrm{~m}^{3}\right]$ is the volume of a monomer. It is noticeable that higher saturation ratios and temperatures allow smaller droplets to be stable. Equation (6) thus gives the rate of creation of particles of size $d_{p}^{*}$ at a given thermodynamic state. A full analysis of the derivation of the terms can be found in $[7,42,43]$.

The wide use of CNT in the analysis of nucleation may be attributed to the availability of data for many substances with regards to their macroscopic thermodynamic properties $\left(\sigma, \rho_{l}, S\right)$ and the availability of critical 
supersaturation [8] values. However, the popularity of CNT has also lead to numerous heuristic expressions for these properties in the literature, of which a selection (including these used in the present work) are presented in Table 1. A small fluctuation in any of these macroscopic parameters may lead to a difference of several orders of magnitude in the nucleation rates.

Table 1: DBP condensation properties

\begin{tabular}{|c|c|}
\hline$M=278.35 \mathrm{~kg} / \mathrm{kmol}$ & ref. $[44]^{*}$ \\
\hline$\rho_{l}=1063-0.826(T-273) \mathrm{kg} / \mathrm{m}^{3}$ & ref. $[10,12,22]^{*}$ \\
\hline$\rho_{l}=1049-0.67\left(T-T_{o}\right) \mathrm{kg} / \mathrm{m}^{3}$ & ref. $[4,45]$ \\
\hline$P_{\text {sat }}=\exp \left(16.27-\frac{5099}{T-109.51}\right) \mathrm{mmHg}$ & ref. $[12]$ \\
\hline$P_{\text {sat }}=\exp \left(16.27-\frac{5099}{T-109.65}\right) \mathrm{mmHg}$ & ref.[14] \\
\hline$P_{\text {sat }}=10^{7.065-1666 / T-547700 / T^{2}} \mathrm{mmHg}$ & ref. $[22,45]^{*}$ \\
\hline$P_{\text {sat }}=10^{-4501 / T+12.88} \mathrm{~Pa}$ & ref. $[4,46]$ \\
\hline$P_{\text {sat }}=\exp \left(160.25-\frac{16941}{T}-19.254 \ln T+\frac{6.6324 \times 10^{-6}}{T^{2}}\right) \mathrm{mmHg}$ & ref. $[44]$ \\
\hline$\sigma=(35.3-0.0863(T-273)) \times 10^{-3} \mathrm{~N} / \mathrm{m}$ & ref.[10, 12] \\
\hline$\sigma=(33.93-0.0894(T-293.15)) \times 10^{-3} \mathrm{~N} / \mathrm{m}$ & $\operatorname{ref} .[4,45]$ \\
\hline$\sigma=0.059663 \times\left(1-\frac{T}{T_{\text {crit }}}\right)^{1.2457} \mathrm{~N} / \mathrm{m}$ & ref. $[44]$ \\
\hline$\sigma=1.04(35.3-0.2(T-273)) \times 10^{-3} \mathrm{~N} / \mathrm{m}$ & ref. $[22]$ \\
\hline$\sigma=1.0(35.3-0.13(T-273)) \times 10^{-3} \mathrm{~N} / \mathrm{m}$ & $*$ \\
\hline
\end{tabular}

*Unless stated otherwise, these are the expressions used in this work

Additionally there have been a great number of modifications to the CNT, 
and therefore to the expression for $\dot{I}$. Lothe and Pound [47] considered transitional and rotational degrees of freedom contributing to the free energy $\Delta G$, increasing nucleation rates by 17 orders of magnitude. Reiss [48] argued that using the experimental values for surface tension variation incorporates these degrees of freedom, since the partial derivative of the Gibbs free energy with respect to surface area is the surface tension. In an attempt to formulate a self-consistent theory, Girshick et al. [49] proposed a correction factor to the nucleation rate such that $\dot{I}_{s c}=\dot{I} \times \exp (\theta) / S$, where $\theta=\sigma s_{m} / k_{B} T$ is a dimensionless surface energy and $s_{m}$ is the surface area of a monomer, such that for a monomer $\Delta G=0$.

Several authors $[50,51]$ modified the nucleation rate by altering the energy barrier to include the differences between the surface free energy of a cluster and of a liquid droplet. They included the self-consistent theorydriven parameter $1 / S$ in the nucleation term but added a contribution of additional degrees of freedom and a curvature dependence of the surface energy on local thermodynamic properties. Hale [52] assumed a linear variation of the surface tension with respect to temperature, and suggested that critical supersaturation can be calculated with the use of a universal constant that measures the excess entropy of a molecule's surface. Granasy [53] suggested a parametrization where both enthalpy and density profiles of the droplets are shifted by a constant amount which is independent of droplet size. In this way he obtained a curvature correction without having to re-establish relationships for the macroscopic thermodynamic parameters. A thorough review of the literature describing the various corrections to the CNT can be found in the work of Laaksonen [8]. 
From the above discussion it can be concluded that there are a number of problems posed by the CNT. In principle it has shown good agreement with experimental results, but has always been subject to modifications. It can be argued that the parameter with the least certainty is the surface tension (rather than the critical saturation temperature or pressure). A correct treatment and calculation of $\sigma$ may mitigate the effects of additional degrees of freedom on the activation energy required for nucleation, along with material-based inconsistencies. Following a parameter analysis, and similarly to the work of Garmony and Mastorakos [22], the surface tension was modified to match the order of magnitude of nucleation rates observed for this particular test case (see Table 1).

\subsection{Turbulent Coupling}

A number of studies $[4,20,22,54]$ have shown that the calculation of nucleation rates from mean values leads to erroneous results, due to the high non-linearity of Eqn. (6). The test case studied in this work has been simulated using a number of methods which stem from population balance modelling, and these are outlined below.

The evolution of the PSD in turbulent flows can be described mathematically using the Population Balance Equation (PBE). The PBE is an equation for the evolution in space and time of the particle number density, $\mathcal{N}\left(d_{p} ; \mathbf{x}, t\right)$, for a given particle diameter $d_{p}$. The integration of $\mathcal{N}$ over all possible diameters gives the total number of particles per unit volume, $N_{p}$. An in-depth investigation of the PBE exceeds the purpose of this work; the interested reader may refer to the review of Rigopoulos [55].

Garmony and Mastorakos [22] implemented a two-dimensional RANS us- 
ing a stochastic fields method for the solution of the PBE. The nucleation rate was considered as a source term, adding newly formed particles of size $d_{p}^{*}$ to the equivalent class of the number density equation. A moment-based approach [56] was used, where an Itô equivalent Stochastic Partial Differential Equation was solved for the evolution of five scalars (temperature, concentration and the first three moments of the PBE) and a log-normal distribution was presumed for the unresolved moments.

Veroli and Rigopoulos [4] followed a PBE-PDF approach [57]. They transport a one-point joint-PDF of temperature, concentration and a set of discretized number densities. The RANS-PDF simulation was solved using a Lagrangian Monte Carlo method [58].

Zhou and Chan [9] proposed an Equivalent Mean Nucleation Method (EMNM) coupled with an LES solution for the jet flow. Along with the uniform phase solution, DBP concentration and temperature distributions were solved using LES (assuming $L e=1$ ). The EMNM splits the nucleation equation (6) into its exponential and pre-exponential terms. The pre-exponential term, as well as the surface tension, density, and saturation ratio, varies much slower with temperature than the exponentiated function. These values are assummed constant within the narrow temperature range over which nucleation takes place. By integrating the product of the strongly-dependent exponential term with a temperature PDF, the temporal fluctuations are taken into account. The EMNM assumes that due to the sensitivity and rapid nature of nucleation only a narrow temperature range needs to be integrated over. The mean particle concentration was assumed to be independent of fluctuations in the source term, and on that basis the time-averaged nucle- 
ation rate was used for all time-steps [9].

In the present work, nucleation is modelled using the LES spray-PDF equation, of which the derivation can be found in Jones et al. [33]:

$$
\frac{\partial \tilde{\mathbb{P}}}{\partial t}+\frac{\partial}{\partial \mathbf{X}_{p}}\left(U_{p} \tilde{\mathbb{P}}\right)+\frac{\partial}{\partial \mathbf{V}_{p}}\left(A_{p} \tilde{\mathbb{P}}\right)+\frac{\partial}{\partial N_{p}}\left(\dot{N}_{p} \tilde{\mathbb{P}}\right)=0
$$

The spray-PDF $\tilde{\mathbb{P}}=\tilde{\mathbb{P}}\left(\mathbf{V}_{p} ; \mathbf{X}_{p} ; N_{p}, t\right)$ describes the joint probability of the particle phase state vector $\boldsymbol{\Phi}$ to retrieve a specific value at a given time. Here, the state vector includes seven variables: the three dimensional velocity $\mathbf{V}_{p}$ and position $\mathbf{X}_{p}$, and the number concentration $N_{p}$. The rates $U_{p}$ and $A_{p}$ represent the conditional mean particle velocity and acceleration as functions of time and space. These quantities can be retrieved by ensemble-averaging the path equations (1) and (2) conditioned upon the local state vector describing the process. Lower-case letters are used to describe the Lagrangian realizations $n_{p}, \mathbf{x}_{p}$ and $\mathbf{v}_{p}$ of the (upper-case) equivalent random variables.

If only nucleation is considered (i.e. neglecting fragmentation and aggregation), every formed particle of size $d_{p}^{*}$ is represented by a single numerical parcel which has a constant number concentration, $n_{p}$, throughout its motion. Therefore for the equivalent path and Eulerian formulations we have:

$$
\begin{aligned}
& \frac{d n_{p}}{d t}=\mathrm{i} \\
& \dot{N}_{p}=\dot{I}-\mathbf{U}_{f} \frac{\partial N_{p}}{\partial \mathbf{x}}
\end{aligned}
$$

where the Lagrangian rate ' $i$ ' is used to differentiate from the Eulerian nucleation rate $\dot{I}$. Note that the convective effects in (11) are included implicitly in the particle path equations (1) and (2), as the rate in (10) is Lagrangian and represents a total derivative. Essentially the Lagrangian rates of change 
of concentration are conditioned upon the event of a particle being at a given location $\mathrm{x}$ of the Eulerian domain at a time $t$.

If physical particles are used the spray-PDF is only a function of $\mathbf{X}_{p}$, $\mathbf{V}_{p}$ and time $t$. The addition of a particle by nucleation simply adds a path (realization) to the trajectories of position (1) and acceleration (2). If notional particles (i.e. stochastic parcels) are used, in which the real particle number concentration can vary from and include zero, equation (10) must be solved as well.

If physical particles are used, the Eulerian variable $N_{p}(\mathbf{x}, t)$ representing particle concentration in a given position and time is reconstructed from the instantaneous sum of the individual paths conditioned upon their location $\mathbf{x}$. Once temporally averaged, $\left\langle N_{p}(\mathbf{x}, t)\right\rangle_{t}$ gives the mean particle concentration at a given location of the Eulerian domain. In contrast, if the number density is included in the particle state vector, the ensemble average of the number density paths of the stochastic parcels would give $\mathcal{N}\left(d_{p} ; \mathbf{x}, t\right)$, and the conditional ensemble average rate of change of the individual paths would be required to evaluate $\dot{\mathcal{N}}\left(d_{p} ; \mathbf{x}, t\right)$.

Using equation (9), the PSD can be readily retrieved at any location in the domain by constructing a histogram of the number of particles at that location as a function of their size. This is equivalent to including the particle size in the spray-PDF formulation and calculating the joint probability of particle concentration and diameter by eliminating the remaining state vector variables, where $N_{\text {tot }}$ is the total particle number concentration in the domain:

$$
\mathcal{N}\left(d_{p}, t\right)=N_{\text {tot }} \int_{\mathbf{X}_{p}} \int_{\mathbf{V}_{p}} \mathbb{P}\left(\mathbf{V}_{p} ; \mathbf{X}_{p} ; d_{p} ; N_{p}, t\right) d \mathbf{X}_{p} d \mathbf{V}_{p}
$$

Multiplication by $N_{t o t}$ is required as the spray-pdf has a unit integral whereas 
the integral of the number density should be equal to the total number of particles per unit volume.

\subsubsection{Probabilistic approach to nucleation}

The proposed method takes advantage of the ability of LES to provide instantaneous values of the uniform phase velocities, temperatures and concentrations, thus enabling the calculation of an instantaneous nucleation rate. This avoids the errors of several orders of magnitude that may result where nucleation rates are calculated from mean flow quantities. Similarly, one may expect that nucleation rates calculated from temporal-mean values, such as the ones used in the aforementioned studies, result in a level of lost information regarding instantaneous rates. Before describing the method used in the present work, the drawbacks of other approaches are presented to illustrate the need for an alternative methodology.

The first consideration relates to the framework in which the nucleation rate should be calculated: namely, should the rate be transported by Eq. (10), or calculated on a purely Eulerian-node basis Eq. (11). To illustrate this, consider a 3 -cell grid of a $3 \times 1 \mathrm{~m}$ domain with a constant velocity inflow

plane and thermodynamic conditions giving a constant rate $\dot{I}=0.4 \# \mathrm{~s}^{-1} /$ cell, as in Fig. 2.

If the time-step is $\Delta t=1 \mathrm{~s}$, the grid-spacing is $\Delta=1 \mathrm{~m}$, and the velocity is $U_{f}=1 \mathrm{~m} / \mathrm{s}$, then for the whole domain a single particle should be created after every time-step. Nucleation is a jump process, however, suggesting that a particle can either be created or not. On a cell basis, after a single time-step, 0.4 particles are created; therefore no physical particles are added to any of the cells. Arguably one may avoid this by creating 


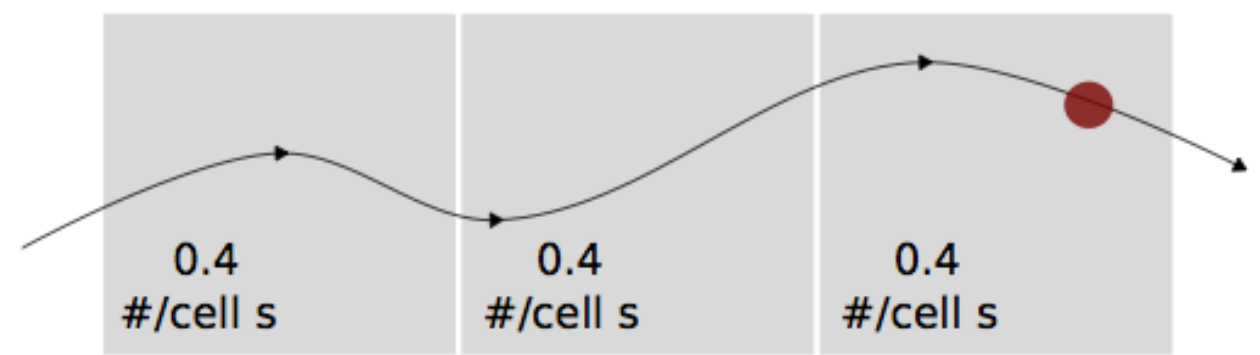

Figure 2: If three cells have a constant nucleation rate of 0.4 particles per cell per second, the whole domain should produce one particle every $0.83 \mathrm{~s}$. However due to the discrete nature of particles, each cell creates 0.4 particles i.e. no physical particles. If fluid enters from the left with a velocity of $U_{f}=1 \mathrm{~m} / \mathrm{s}$ and a cumulative nucleation term is considered then as transported from the first cell to the last with a time-step of $1 s$ a single particle is created in the last cell.

an accumulation parameter for every cell and adding one particle to every cell after $3 \mathrm{~s}$. However, using an accumulation parameter implicitly assumes time-averaging of the instantaneous nucleation rate in cases where $\dot{I}$ is not constant in a cell.

By considering an 'accumulated', i.e. a transported source term 'i', the above problem can be surpassed. The nucleation rate is transported such that after the first time-step 0.4 notional particles are created in the first cell; in the next time-step, after moving to the next cell, 0.8 notional particles are created; and in the final time-step a physical particle is added into the last cell. Such a transported approach creates two additional issues. Firstly, a bias is created towards particle creation in the final cell, even though in theory the nucleation rates are constant throughout the domain (suggesting that the same number of particles are created everywhere). 
Secondly, a transported approach creates a dependence of particle creation on the relation between flow-through time and nucleation residence time. To illustrate this, consider the same example as above (where the local concentrations and temperatures give a constant nucleation rate for every cell of 0.4 particles per second), but with a reduced time-step of $0.5 \mathrm{~s}$ and a velocity of $U_{f}=2 \mathrm{~m} / \mathrm{s}$ (Fig. 3). By transporting a notional particle which accumulates 'substance' in every cell, by the end of the domain 0.6 notional particles are created; therefore no physical particles are added. For a fixed CFL number, the reduced time-step is equivalent to a faster-moving flow. If one tracks the motion of a control volume moving at this speed and with constant thermodynamic conditions, the residence time of nucleation $\tau_{n u c}$ (i.e. one particle per cell in $2.5 \mathrm{~s}$ ) must be less than or equal to the flow-through time $\tau_{\text {flow }}=3 \Delta / U_{f}$ for a particle to be created. For the present case this tracking would result in no particles being created even after $t>\tau_{\text {nuc }}$, even though the local thermodynamic conditions should create a particle in the domain approximately every $0.83 \mathrm{~s}$.

In hindsight, CNT stems from a thermodynamic equilibrium theory where the saturation and concentration levels are known for a closed system, enabling the calculation of a nucleation rate. Therefore the main question is how to treat such an equilibrium theory in non-stationary turbulent flows.

From the example illustrated in Fig. 2, one may observe that the derivation of the nucleation rate from an equilibrium theory involves a lack of information, since it is a predicted mean. Knowing the overall nucleation rate for the domain we expect a particle to be created within $0.83 \mathrm{~s}$, but there is no knowledge in terms of when it will occur, and in the case of the 


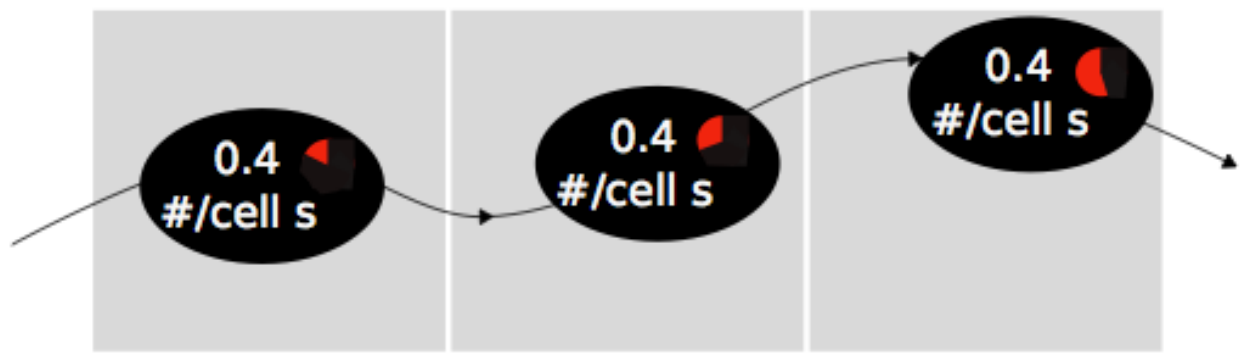

Figure 3: Consider three cells which have a constant nucleation rate of 0.4 particles per cell per second, the time-step is $0.5 \mathrm{~s}$, and the inflow plane velocity is $U_{f}=2 \mathrm{~m} / \mathrm{s}$. A transported nucleation rate approach creates a bias towards particle creation in the final cell and a dependence between flow-through time and nucleation residence time.

transported example (figure 3) where. The nucleation rate gives a sufficient condition for a number of particles to be formed within a volume in a given time, but it does not provide a conditionality for this creation.

The solution presented in this work assumes that the nucleation rate can be treated as the transient probability of a stable embryo being formed, and not just a steady-state condition for its creation. This assumption agrees well with the suggestion of McDonald [59], that nucleation is intrinsically a probabilistic process. The inverse of the nucleation rate can be considered as the average time required for a particle to nucleate. By calculating the instantaneous nucleation rate for each time-step at each node of the Eulerian domain, one may calculate the residence time required for a single particle to be created in the vicinity of the node:

$$
\tau_{n u c}=\frac{1}{\dot{I} \times V_{\text {cell }}},
$$

where $V_{\text {cell }}$ is the volume of the cell. Here, we assume that the time $\tau_{\text {nuc }}$ represents an event with unit probability $\mathcal{P}_{\text {nuc }}\left(\tau_{\text {nuc }}\right)=1.0$, i.e. a single 
particle will be created in time $t=\tau_{n u c}$. The probability of a particle being created in the interval $0 \leq t \leq \tau_{n u c}$ is assumed to increase linearly with $t$ :

$$
\mathcal{P}_{\text {nuc }}(t)=\frac{t}{\tau_{\text {nuc }}}
$$

$\mathcal{P}_{n u c}$ is a Cumulative Distribution Function (CDF), and provides the probability of an event to occur at any time within the interval $0 \rightarrow t$. The linear increase of the CDF suggests a uniform PDF of value $d \mathcal{P}_{n u c} / d t=1 / \tau_{n u c}$ in the range $0 \leq t \leq \tau_{\text {nuc }}$. It must be stressed that nucleation is an activated process and not a probability per se and that the CNT gives the steady-state transition rate (in this case DBP vapour $\rightarrow$ liquid). In stochastic theory, the Mean First-Passage Time (MFPT) is defined as the average time elapsed until a certain system leaves a prescribed domain for the first time [60]. The MFPT as a function of current and initial state has a characteristic sigmoidal shape [61]. The inverse of the steady state MFPT is directly related to the transition rate $\dot{I}$ and this is the scale we term $\tau_{n u c}$. For simplicity, we assume a linear increase in the probability (i.e. uniform pdf) of the activation barrier crossing in the range of $0<t<\tau_{n u c}$. However, the sigmoidal shape suggested by the MFPT-theory in the work of Wedekind and co-workers could be better approximated, for example, using a Poisson distribution.

Using the definition of equation (14) the nucleation jump term is modelled as a particle injection into the domain, and the particle is thereafter transported as described by Eqs. (1) and (2). The injection process can be summarized as follows:

- The probability of a particle being created in a cell in one time-step of the simulation is calculated using equation (14). Recall that the 
nucleation residence time is a function of space and time $\tau_{n u c}(\mathbf{x}, t)$.

- If $\mathcal{P}_{\text {nuc }}>1$ the simulation time-step is reduced.

- A gambling process is performed using a random number $R n$, sampled from a uniform distribution in the range $0 \leq R n \leq 1$ to decide whether to inject a particle:

$$
\text { Inject }= \begin{cases}\text { True, } & \text { if } R n \leq \frac{\Delta t}{\tau_{n u c}} \\ \text { False, } & \text { otherwise }\end{cases}
$$

- If (15) is true a particle with size equal to the nucleation diameter $d_{p}^{*}$ at that location is injected.

- The particle injection position is random (within the cell boundaries), with a uniform probability distribution.

- The velocity and temperature of the newly-formed particle are linearly interpolated from the cell-centred (Eulerian) values of the surrounding cells.

The above method allows for the calculation and direct use of instantaneous nucleation rates, instead of mean quantities, without the addition of any modeling parameters. In addition, by recalculating the residence time in every time-step, positional biases of the type mentioned in the examples of Figs. 2 and 3 are removed. The method can be readily applied in the Lagrangian framework using an SDE described by a Levy jump process, viz:

$$
\frac{d n_{p}}{d t}=\mathrm{i}=\mathbf{J}[\mathbf{x}(t), t] \delta N_{d t}^{j, \tau_{n u c}}
$$


where $\mathbf{J}$ is a scaling tensor representative of the local nucleation rate i. The increment $\delta N_{d t}^{j, \tau_{n u c}}$ is the $j^{\text {th }}$ component of a stochastic counting ${ }^{1}$ process with time increments $d t$ and a mean rate $\tau_{n u c}$, which usually follows a Poisson distribution. The advantage of a Lagrangian description is that the exact location of nucleation is retrieved, since the species, temperature, and pressure are transported (or interpolated) at the exact location of the notional parcel.

For the particular test case studied, the residence time for a single cell with a mean nucleation rate of $10^{11} \# \mathrm{~m}^{3} \mathrm{~s}$ (equivalent to the maximum observed nucleation rate, so as to consider the most conservative scenario) and a cell volume of $\Delta^{3}$ is of the order of $1 \mathrm{~s}$, whereas the time-step widths are of the order of $10^{-6} \mathrm{~s}$ based on the maximum flow velocity, cell size, and a $\mathrm{CFL}=0.3$. This suggests that the simulation should run for at least $10^{6}$ time-steps for one particle to be seen in every cell with this mean nucleation rate. Therefore, in this study, the nucleation rate is modelled in an Eulerian framework and the parcels represent physical particles which are subsequently transported in a Lagrangian framework using equations (2) and (1).

The time convergence of the concentration statistics is promoted by a multiplying factor, which essentially increases the sampling time. This factor (here $f_{r}=100$ ) is multiplied with the LES time-step such that additional particles are created for a single nucleation event without influencing the thermodynamic parameters of the formation. Therefore, if the condition of

\footnotetext{
${ }^{1}$ The term 'counting' is used to clarify that the set of realizable values is discontinuous.
} 
(15) is true, $f_{r}$ particles are injected which are subsequently tracked individually. Approximately 1 million particles were present in the domain at an instant in time. The reconstruction of the Eulerian concentration field from the Lagrangian particles is then divided by $f_{r}$ at every nodal point of the field. Simulations are run both with and without the use of the multiplying factor, and the results are compared in section 4.3.

\subsubsection{Modelling the unresolved scales}

The time-averaging of nucleation rate may lead to both implementation errors and a problematic description of equation (6) when applied to nonstationary flow conditions. LES filtering may be described as the convolution of a function $g(\mathbf{x}, t)$ with a filtering kernel $G_{\Delta}$ over a given volume $V$ :

$$
\tilde{g}(\mathbf{x}, t)=\int_{V} g(\mathbf{x}, t) G_{\Delta}(\mathbf{x}, t) d V,
$$

where $\tilde{g}$ is an LES filtered quantity. LES filtering introduces unresolved sub grid-scale fluctuations of the transported quantities. These fluctuations may influence the nucleation rates, depending on the level of concentration and temperature information resolved by the LES (and consequently the level of lost information regarding fluctuations) within a cell.

Recently, Fager et al. [62], investigated the relative effects of sub-grid scales on the nucleation rate by a posteriori filtering of DNS of turbulent jet They concluded that the filtered nucleation rate is under-predicted in inner-regions of the jet and over-predicted in the outer regions. Similarly, Murfield and Garrick [25] found that the overall sub-grid effect is to increase the values of nucleation when these were high, and to decrease them when they were low. 
For $L e=1$, a single scalar $\tilde{\xi}$ can be defined from the normalized temperature or vapor concentration, viz:

$$
\tilde{\xi}=\frac{\tilde{T}-T_{c f}}{T_{j e t}-T_{c f}}=\frac{\tilde{X}-X_{c f}}{X_{j e t}-X_{c f}},
$$

where the subscript $c f$ refers to the co-flow initial/inlet conditions. The tilde

corresponds to the LES-filtered value of the defined scalar $\tilde{\xi}$, temperature field $\tilde{T}$, and DBP concentration $\tilde{X}$. The nucleation rate can therefore be written as $\dot{I}(T, X) \approx \dot{I}(\xi)$, while its filtered equivalent is $\widetilde{\dot{I}(\xi)}$. However:

$$
\widetilde{\dot{I}(\xi)} \neq \dot{I}(\tilde{\xi})
$$

The inequality of equation (19) implies that a model is needed. The models employed here are a presumed $\beta$-PDF and a source expansion approach outlined below:

\section{A. $\beta-P D F$}

The scalar $\mathrm{PDF} f_{\xi}(\psi ; \mathbf{x}, t)$ represents the distribution of the realizations $\psi$ of the scalar $\xi$ at a given position $\mathbf{x}$ and time $t$ [63]. Note that in the context of LES the scalar PDF is also termed the filter density function; the term PDF will be used here for consistency. For a complete review of the derivation and applications of the scalar PDF the interested reader may refer to $[64,65,66]$.

One may retrieve the filtered-average source term at a given location from its convolution with $f_{\xi}$ :

$$
\widetilde{\dot{I}(\xi)}=\int_{0}^{1} \dot{I}(\psi) f_{\xi}(\psi ; \mathbf{x}, t) d \psi
$$


If the distribution $f_{\xi}$ is known, the filtered nucleation rate can be directly evaluated.

There are two approaches for the use of the scalar PDF. The first is the 'transported PDF' approach, in which there is no assumption made regarding the shape of the distribution. The transported PDF method is therefore capable of capturing inhomogeneities, but suffers from an increased computational expense. The second method has a significant advantage in computational time by making an a priori assumption for the shape of the PDF, of which the most popular is the $\beta$-PDF (but other functions are also suitable such as this of Floyd et al. [67]). The presumed PDF method has been used in a number of precipitation studies $[68,69]$. The $\beta$-PDF for the sub-grid distribution of a passive scalar has shown good agreement with experimental data and a priori studies in a range of applications including confined jets [70], turbulent mixing [71] among others [72, 73, 74]. For its computational efficiency and adequate description of the SGS scalar distribution, the univariate $\beta$-PDF (21) is chosen:

$$
f_{\xi}(\psi ; \mathbf{x}, t)=\frac{\Gamma\left(\beta_{1}+\beta_{2}\right)}{\Gamma\left(\beta_{1}\right) \Gamma\left(\beta_{2}\right)}\left[\psi^{\beta_{1}-1}(1-\psi)^{\beta_{2}-1}\right],
$$

where $\Gamma$ is the gamma function. The shape parameters $\beta_{1}$ and $\beta_{2}$ are evaluated using the first two moments of the distribution, i.e. the mean $\tilde{\xi}$ and variance $\widetilde{\xi^{\prime \prime}}$, see [75]. The mean $\tilde{\xi}$ is derived from the evolution of the temperature (or concentration field) making use of equation (20). The variance $\widetilde{\xi^{\prime \prime} 2}$ is calculated using the proposed method of Pierce and Moin [76], as used in $[77,78]$ :

$$
\widetilde{\xi^{\prime \prime}}=C_{\xi} \Delta^{2}|\nabla \tilde{\xi}|^{2}
$$


with a model constant of $C_{\xi}=0.1$ as proposed by Branley and Jones [77]. The SGS scalar distribution and derived filtered source terms are tabulated for all combinations of means $\tilde{\xi}$ and variances $\widetilde{\xi^{\prime 2}}$ to minimize computational time.

\section{B. Source Expansion}

An alternative method for the evaluation of the filtered source term has been proposed by Vogiatzaki and Navarro-Martinez [79], and is implemented here in the context of nucleation. This approach stems from a Taylor series expansion of the nucleation rate about the filtered scalar $\tilde{\xi}$ :

$$
\dot{I}(\xi)=\dot{I}(\tilde{\xi})+\left.\frac{\partial \dot{I}(\xi)}{\partial \xi}\right|_{\tilde{\xi}} \frac{\partial \xi}{\partial x_{i}} \delta x_{i}+\left.\frac{1}{2} \frac{\partial^{2} \dot{I}(\xi)}{\partial \xi^{2}}\right|_{\tilde{\xi}} \frac{\partial \xi}{\partial x_{i}} \frac{\partial \xi}{\partial x_{j}} \delta x_{i} \delta x_{j}+\ldots
$$

where the variation of the scalar space to spatial space is related by $\delta \xi=$ $\delta x_{i} \partial \xi / \partial x_{i}$

Equation (23) is subsequently filtered. Assuming that spatial gradients and the vector $\delta \mathbf{x}$ are uncorrelated (i.e. $\widetilde{\delta \mathbf{x}^{m} g} \approx \widetilde{\delta \mathbf{x}^{m}} \tilde{g}$ ), one may define the moments of $\delta \mathbf{x}$ as $M_{m}=\int|\delta \mathbf{x}|^{m} G_{\Delta} d V$. The odd moments of the filtered spatial variation are zero, and for a Gaussian filter the even moment $M_{2}=$ $\Delta^{2} / 12$ [80]. Ignoring high-order terms, the resulting filtered expansion reads:

$$
\widetilde{\dot{I}(\xi)} \approx \widetilde{\dot{I}(\tilde{\xi})}+\left.\frac{\Delta^{2}}{24} \frac{\partial^{2} \dot{I}(\xi)}{\partial \xi^{2}}\right|_{\tilde{\xi}} \frac{\partial \xi}{\partial x_{i}} \frac{\partial \xi}{\partial x_{j}} .
$$

The unfiltered terms can be evaluated directly from the filtered scalar value. The first term of the RHS requires additional modelling since $\widetilde{\dot{I}(\tilde{\xi})} \neq$ $\dot{I}(\tilde{\xi})$. To do so a quadrature method is employed using the trapezoidal rule for 
the weights, $\omega_{i j}$, and the values of the source term from eight neighbouring cells evaluated at the equivalent filtered scalar values, viz:

$$
\widetilde{\dot{I}(\tilde{\xi})}=\omega_{i j} \dot{I}_{i j}(\tilde{\xi})
$$

The last term of the RHS of (25) involves filtering. It is therefore split into its filtered and SGS parts. The sub-grid contribution $\dot{I}_{s g s}$ (by analogy with the scalar variance) is modelled using equation (26) with a constant of proportionality $C_{\text {sgs }}=0.1$ by equivalence to Eqt. (22).

$$
\dot{I}_{s g s}=\widetilde{\frac{\partial \xi}{\partial x_{i}} \frac{\partial \xi}{\partial x_{j}}}-\frac{\partial \tilde{\xi}}{\partial x_{i}} \frac{\partial \tilde{\xi}}{\partial x_{j}} \approx C_{s g s} \frac{\partial \tilde{\xi}}{\partial x_{i}} \frac{\partial \tilde{\xi}}{\partial x_{j}}
$$

Combining equations (24), (25), and (26) results in the following expression for the evaluation of the filtered source term:

$$
\widetilde{\dot{I}(\xi)} \approx \omega_{i j} \dot{I}_{i j}(\tilde{\xi})+\left.\frac{\Delta^{2}}{24} \frac{\partial^{2} \dot{I}(\xi)}{\partial \xi^{2}}\right|_{\tilde{\xi}}\left(1+C_{s g s}\right) \frac{\partial \tilde{\xi}}{\partial x_{i}} \frac{\partial \tilde{\xi}}{\partial x_{j}}
$$

The second derivative of the nucleation rate was derived analytically and tabulated with 10000 bins of the scalar $\xi$ to minimize computational time.

\section{Results}

\subsection{Uniform phase}

Figure 4 shows the instantaneous and mean axial velocity contours of the LES. The jet enters the chamber via an insulated nozzle and mixes with the co-flow of air. After an initial undisturbed region a shear-mixing layer develops and co-flowing air is entrained into the developing jet which facilitates its spreading. 


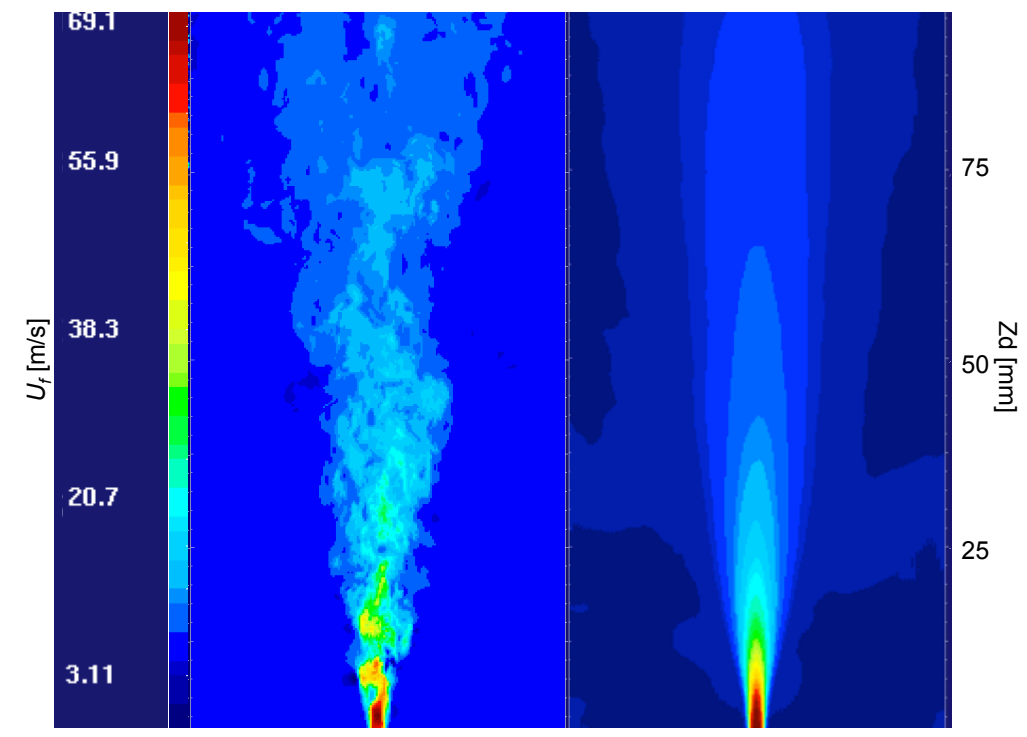

Figure 4: Instantaneous (left) and mean (right) axial velocity contours

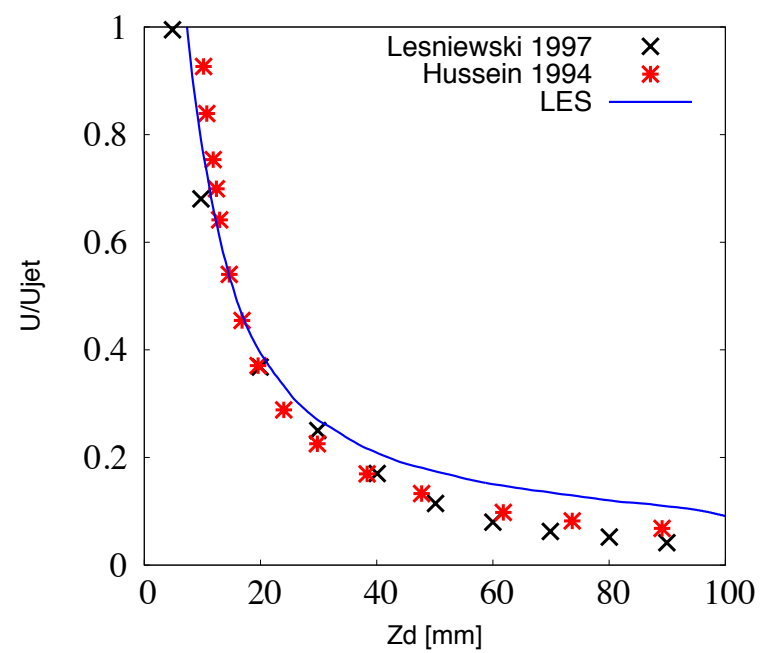

Figure 5: Centerline velocity normalized by the jet velocity along the domain. 


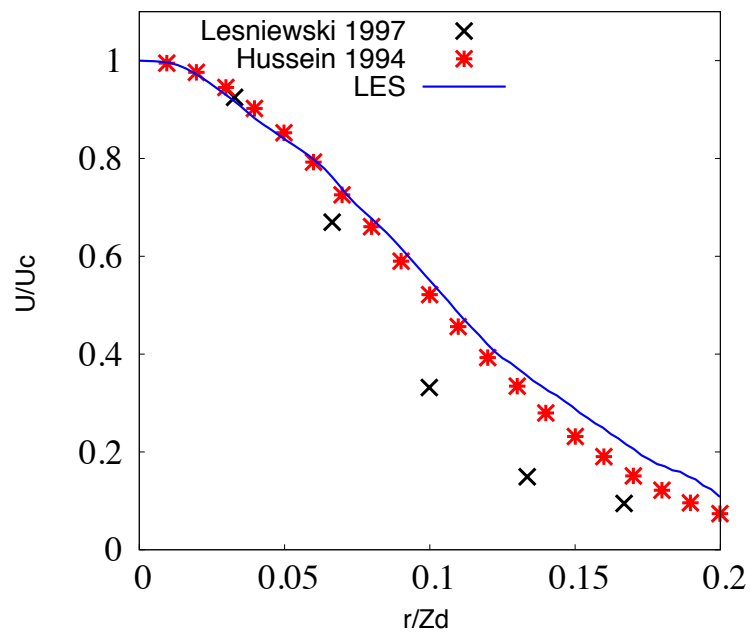

Figure 6: Radial velocity plot normalized by the centerline velocity and axial location. The solution becomes self-similar for mean profiles beyond $Z_{d} / D_{\text {jet }}=30$

The LES solution of the velocity distribution was compared to the experimental data of [21] and the self-similar jet solution of Hussein [18]. Figure 5 depicts the centerline velocity normalized by the jet velocity along the domain. A good agreement is found with the experimental data near the jet outlet, but a slower decay is observed further downstream, which persisted for smaller $\Delta$ and with the use of the dynamic Germano model [81]. A more gradual radial decay (compared to the experimental findings of [21]) was also observed in the LES of Zhou and Chan [9] and the RANS of Veroli and Rigopoulos [4]. It can be seen that the LES results are closer to the self-similar solution than to the experimental data. The radial profile collapses on to a single self-similar solution beyond $Z_{d} / D_{\text {jet }}=30$, as shown in Fig. 6. Hussein [18] observed a similar position at which self-similarity is 


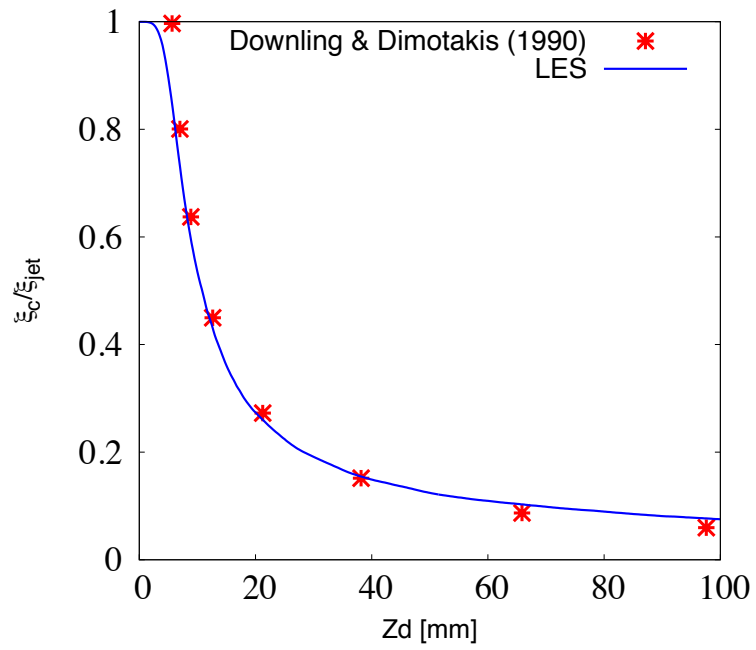

Figure 7: Centerline scalar distribution in axial direction normalized by the initial jet concentration. The points represent the self-similar solution of [82].

reached.

In the experiment, temperature and concentration axial decay was not measured. The self-similar solution for a turbulent jet of [82] is therefore used for comparison with the LES results (see Fig. 7).

\subsection{Nucleation Rate}

As in previous work $[4,22,9]$, the CNT was found to significantly underestimate the observed nucleation rates by six orders of magnitude. A modification to the surface tension similar to the work of Garmony and Mastorakos [22] was implemented to compensate for this discrepancy. Figure 8 shows the variation of the nucleation rate with the scalar $\xi$ for DBP at the reference temperatures of the experiment. The nucleation rate is significant only for a narrow (30\%) range of the values of $\xi$. 


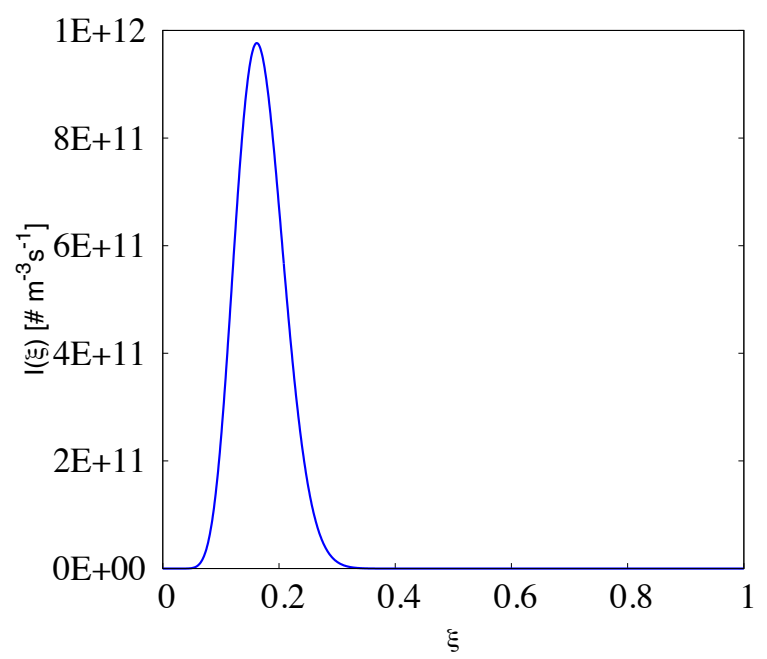

Figure 8: Nucleation rate as a function of the scalar $\xi$.

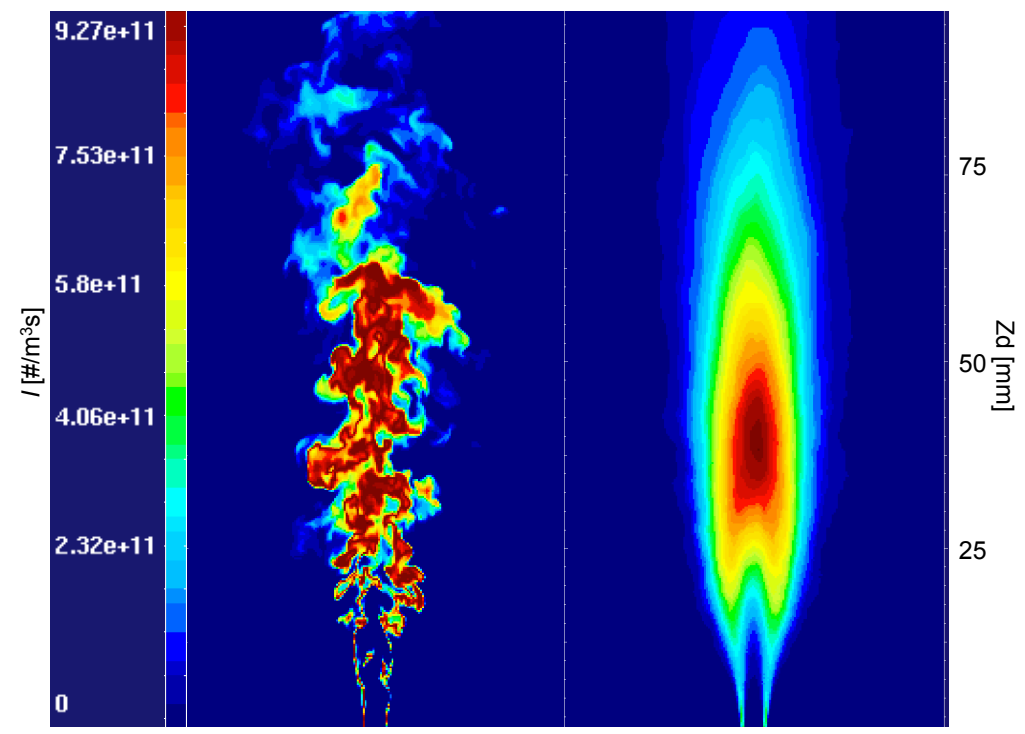

Figure 9: Instantaneous (left) and mean (right) nucleation rate contour plots. The nucleation rate reached a peak of order $10^{11}\left[\# / \mathrm{m}^{3} \mathrm{~s}\right]$. 
Figure 9 shows a snapshot of instantaneous (left) and mean (right) nucleation rates from the LES. From the mean profiles of Fig. 9 it is noticeable that the highest nucleation rates are observed near the centreline between $Z_{d}=[30-50] \mathrm{mm}$. On the other hand, the positions where high nucleation rates occur instantaneously are highly irregular. It should be noted that the mean values shown in Fig. 9 are the time-averaged instantaneous nucleation rates from the LES. These mean values are not calculated from time-averaged scalar concentrations, i.e. the mean nucleation rate from the LES simulation is $\overline{\dot{I}}_{L E S}=\langle\dot{I}(\tilde{\xi})\rangle_{t}$, where $<\cdot>$ denotes averaging over a number of temporal samples. In contrast, for RANS simulations and the LES performed by Zhou and Chan [9], the mean nucleation rate was calculated from the converged mean scalar distribution: i.e. $\overline{\dot{I}}_{R A N S}=\dot{I}(\bar{\xi})$ and $\overline{\dot{I}}_{E M N M}=\dot{I}\left(\langle\tilde{\xi}\rangle_{t}\right)$, respectively, where the same notation is used and $\bar{\xi}$ represents the converged RANS scalar distribution).

An equivalent RANS nucleation rate has been calculated using the values of the LES time-averaged scalar concentration on the centreline (shown in Fig. 7) and is compared to the LES time-averaged nucleation rate. It must be noted that the term equivalent is used because RANS simulations include treatment of the temporal fluctuations. This is therefore a 'naive' RANS of a type that would not be implemented in practice.

Figure 10 (left) depicts that the non-linearity of the nucleation term produces different mean rates for the two cases considered. The use of instantaneous values of the scalar broadens the distribution of the mean rate and slightly shifts the position of the peak downstream. The RANS equivalent case suggests that no particles will form before $Z_{d}=18 \mathrm{~mm}$, which 

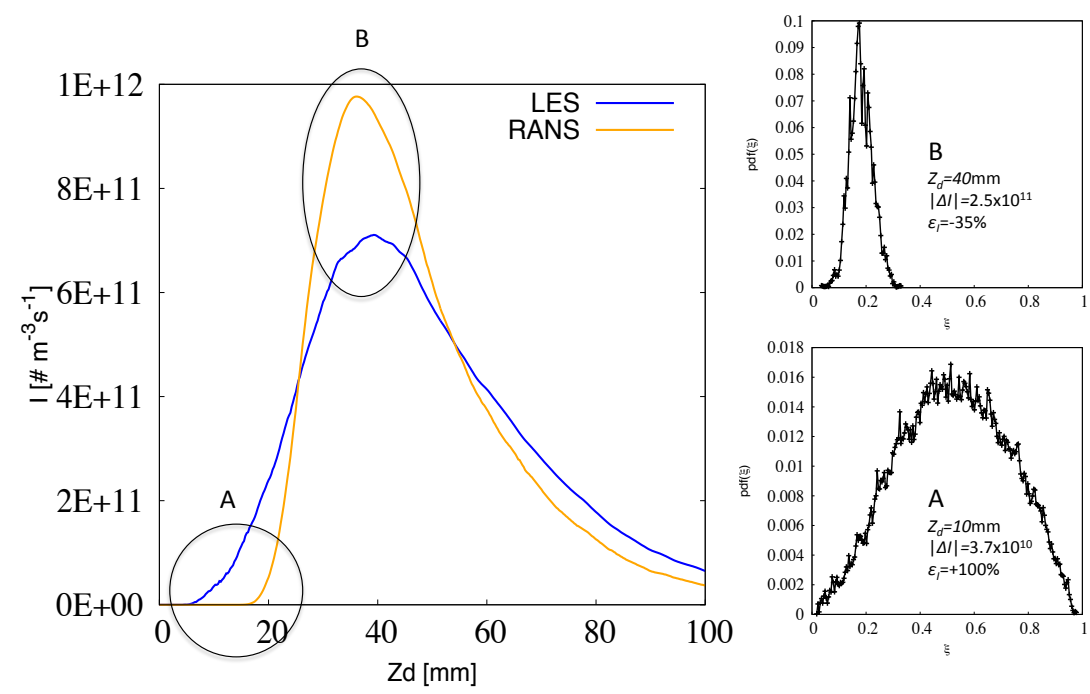

Figure 10: Left: LES nucleation rate calculated from time-average instantaneous values, i.e. $\overline{\dot{I}}_{L E S}=\langle\dot{I}(\tilde{\xi})\rangle_{t}$, versus an equivalent 'naive' RANS where the nucleation rate is calculated using the time-averaged scalar values, i.e. $\overline{\dot{I}}_{e q R A N S}=\dot{I}\left(\langle\tilde{\xi}\rangle_{t}\right)$. Right: $\operatorname{pdf}(\tilde{\xi})$ at the two locations marked A and B. 


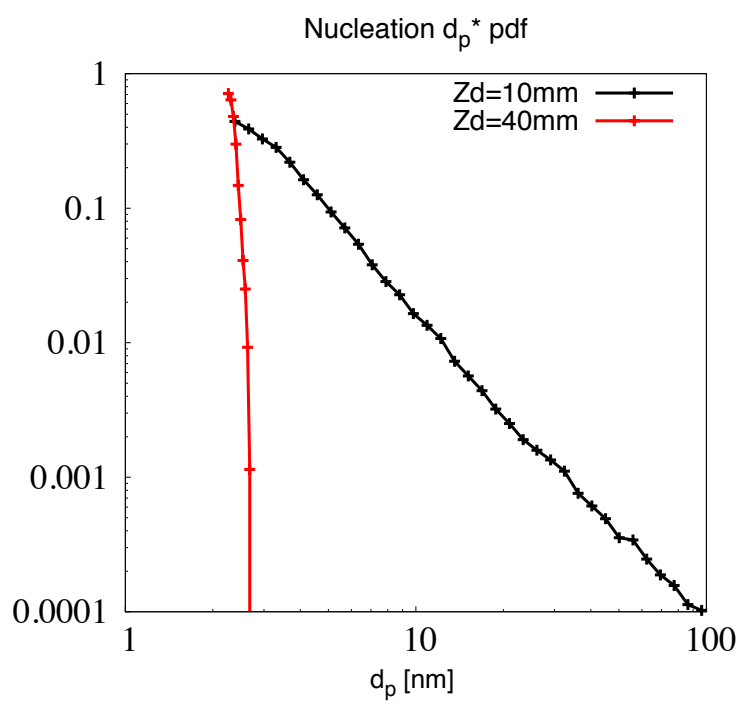

Figure 11: PDF of critical nucleation diameter at two axial locations.

contradicts the experimental findings of [10]. At the two locations marked 'A' and 'B' $\left(Z_{d}=10,40 \mathrm{~mm}\right.$, respectively) the equivalent probability density functions of the LES scalar $\tilde{\xi}$ are shown along with the absolute and relative difference of the two nucleation rates: $\Delta \dot{I}=\overline{\dot{I}}_{L E S}-\overline{\dot{I}}_{e q R A N S}$ and $\epsilon_{\dot{I}}=\Delta \dot{I} / \bar{I}_{L E S}$, respectively. The flapping of the jet near the location 'A' produces a wide temperature pdf and a significant difference in the nucleation rates calculated from the LES and the 'naive' RANS. At location 'B', the pdf is narrower and therefore the relative difference between the two rates is smaller, yet significant (approximately $35 \%$ ).

Figure 11 depicts the distributions of critical nucleation diameter $d_{p}^{*}$, as predicted by the CNT Eqn. (8), at the two axial locations 'A' and 'B'. The range of stable diameters given by the CNT for the instantaneous scalar value at $Z_{d}=10 \mathrm{~mm}$ spans over two orders of magnitude. A sharp delta- 


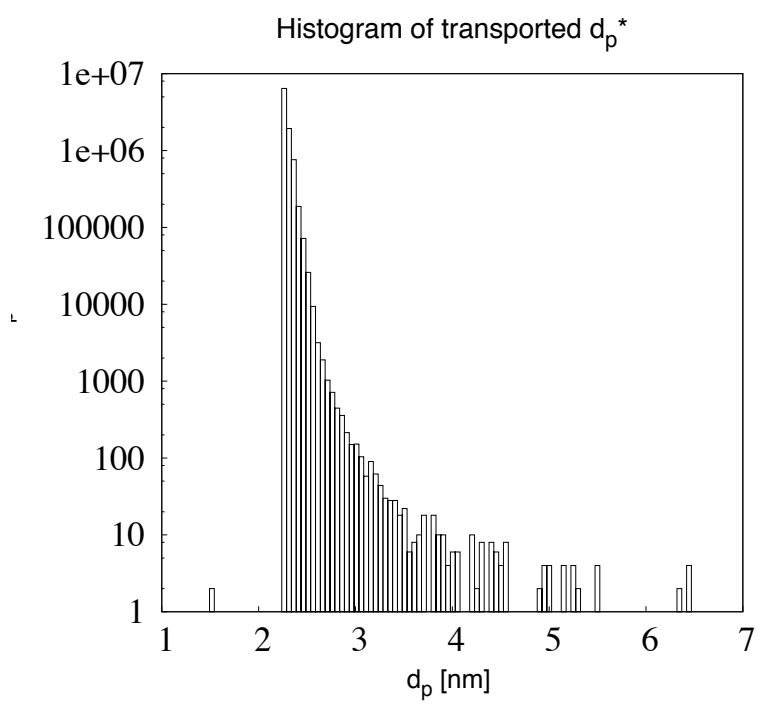

Figure 12: Histogram of formed particles tracked in the simulation.

type function is observed at $Z_{d}=40 \mathrm{~mm}$ as expected from the narrow scalar distribution of the equivalent location shown in Fig. 10.

Figure 12 is a histogram of the formed particles tracked in the simulation at a given instant in time. The majority of the particles nucleated with a diameter of $d_{p} \approx 2.3 \mathrm{~nm}$, which corresponds approximately with the peak diameters of Fig. 11.

Figure 13 shows the scalar fluctuations on the centerline from the LES. In the near-nozzle region the scalar deviates from its mean by up to 0.2 , which is equivalent to a temperature fluctuation of $23 \mathrm{~K}$. This suggests that although the mean concentration may result in insignificant or zero nucleation, instantaneous rates may reach values of the order of $10^{11}\left[\# / \mathrm{m}^{3} \mathrm{~s}\right]$.

Instantaneous data include a higher level of information than mean values, so that LES provides a significant advantage over RANS for this con- 


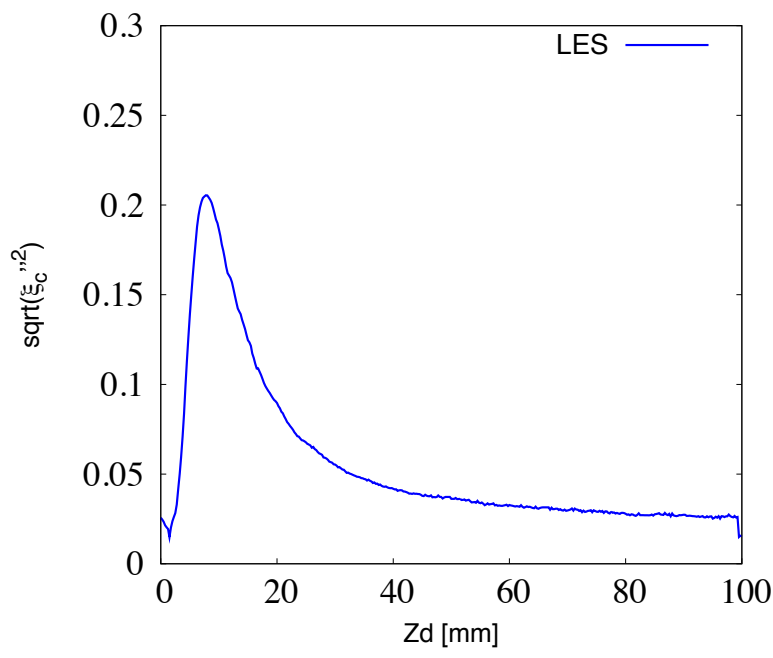

Figure 13: RMS scalar on the centerline.

figuration. Moreover, when nucleation is viewed in conjunction with particle injection the differences between using mean and instantaneous values have been highlighted in the examples of Figures 2 and 3.

To effectively use the additional information of the LES the particle creation must also be based on the local instantaneous conditions. The following section provides an overview of the results of the proposed method for the particle concentrations, and a comparison to the available experimental data. Finally, the effects of the unresolved scales on particle concentrations and nucleation rates are evaluated.

\subsection{Particle Concentration}

Figure 14 shows a snapshot of instantaneous and mean particle number concentrations $N_{c}$ [\# per cell]. These fields are constructed from an ensemble summation over the Lagrangian particles. In a given cell the particle 


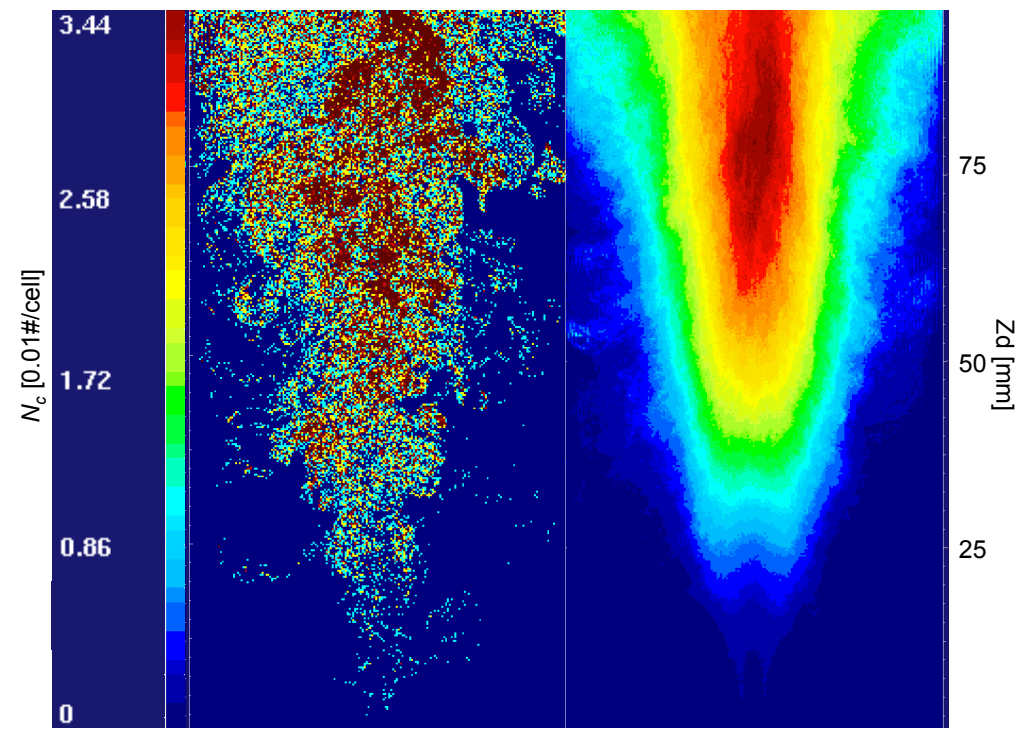

Figure 14: Instantaneous (left) and mean (right) particle concentrations. Note that the mean range varies from $N c=0-0.0365$ [\# per cell], whereas the instantaneous $N c=$ $0-0.15$ [\# per cell] .

number concentration is calculated at every time-step. When compared to the nucleation contours in Fig. 9, one may notice that the position of peak concentration is downstream of the position of the peak formation rate. This is an outcome of the flow-field, i.e. 'cumulative nature' of this experimental set up where particles accumulate as the jet decelerates. At a given axial location, particles are counted using the $\mathrm{CPC}$; however, the exact origin of each nucleation event cannot be determined experimentally. Measurements of particle concentration therefore have a cumulative bias, as particles nucleated elsewhere in the domain are transported by the flow to the position of the counter.

If particles always nucleated with the same initial diameter and constant growth rate one would be able to determine their nucleation location from 
their current size and the required time to reach that size; in a turbulent flow, however, there are fluctuations in the nucleation diameter as well as in the position at which a particle is formed. The cumulative effect in the $\mathrm{CPC}$ measurement becomes one of the primary reasons for which nucleation rates cannot be directly measured in an open system. In contrast, expansion chambers, which are used to measure nucleation rates in a confined volume, allow for a certain residence time (equivalent to the expansion stroke of the piston) before nucleation is quenched (by a slight re-compression of the piston). Nucleation rates can therefore be measured by counting the number of particles produced within the volume of the chamber, in the allowed residence time, under the enforced macroscopic thermodynamic state. In turbulent nucleating jets (and in open systems in their generality) the exact origin of a nucleation event cannot be traced, and hence the formation rate at a given location cannot be determined.

Figure 15 depicts that the use of a multiplying factor to the nucleation rate leads to a more rapid convergence in particle concentration statistics. The flow has correlations which are much shorter than the nucleation residence time, and in conjunction with the results in Fig. 15 it can be argued that the multiplying factor does not adversely influence the nucleation process.

The LES results with no contributions are compared to the experimental results in figures 16 and 17.

Good agreement is found with the experimental results and with the computations of Garmony and Mastorakos [22]. DiVeroli and Rigopoulos [4] underestimated the concentration levels by at least one order of magnitude. It should be noted that the LES underestimates the first particle 


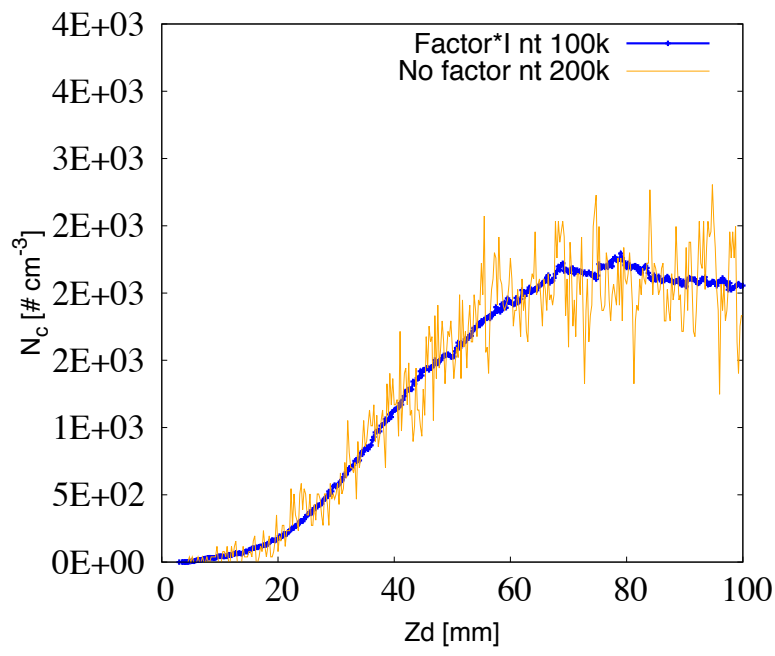

Figure 15: Mean particle concentration along the centerline, with and without a multiplying factor. The results are averaged after 100 and 200 time-steps, respectively.

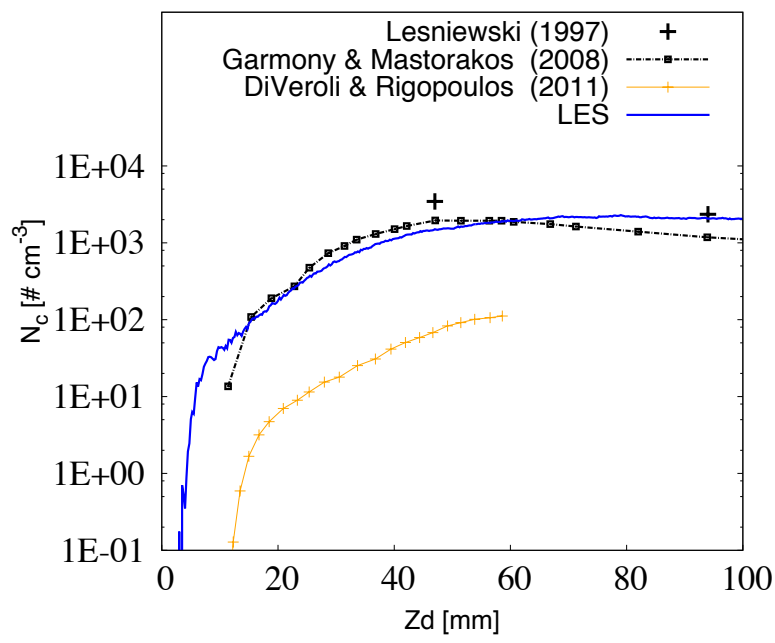

Figure 16: Particle concentration on the centerline in the axial direction. The LES results are compared to the experimental data and two previous numerical solutions for this test case 


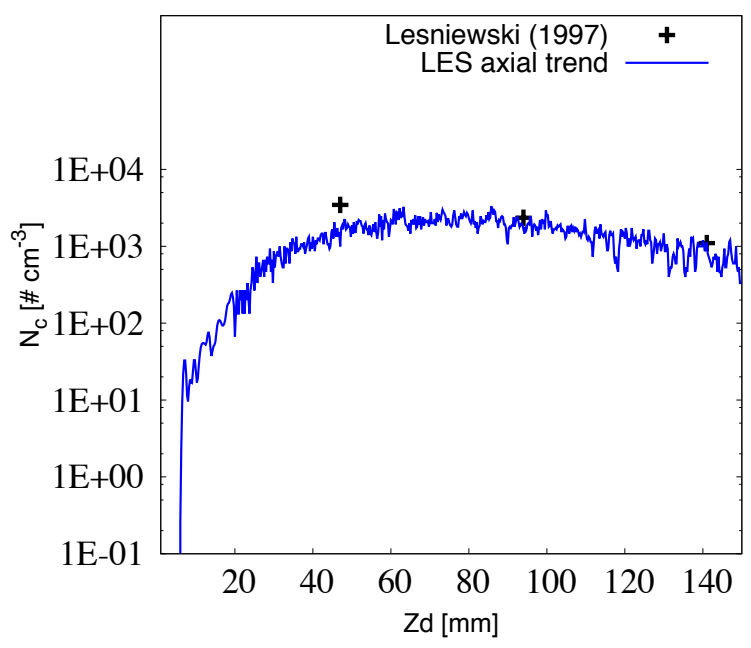

Figure 17: Particle concentration on the centerline in the axial direction. A longer domain was used and a similar decay of particle concentration was found downstream

concentration measured experimentally. However, the axial trend in particle concentration levels is well-captured by the LES further downstream, as shown in Fig. 17. A similar underestimation of the first measured point was reported in the work of Garmony and Mastorakos [22]. The discrepancy may be caused by extra nucleation in the sampling tube: the residence time in the sampling tube leading to the $\mathrm{CPC}$ is around $0.3 \mathrm{~s}$, which may lead to additional nucleation at upstream locations where the DBP vapor concentration is still high. Moreover, the jet flow becomes self-similar beyond $Z_{d} / D_{j e t}=30,(70.5 \mathrm{~mm})$ and therefore vapour concentration and temperature distributions only become self-similar downstream of the first measured point. Therefore a closer examination is required both of nucleation rates and of particle number concentrations. 


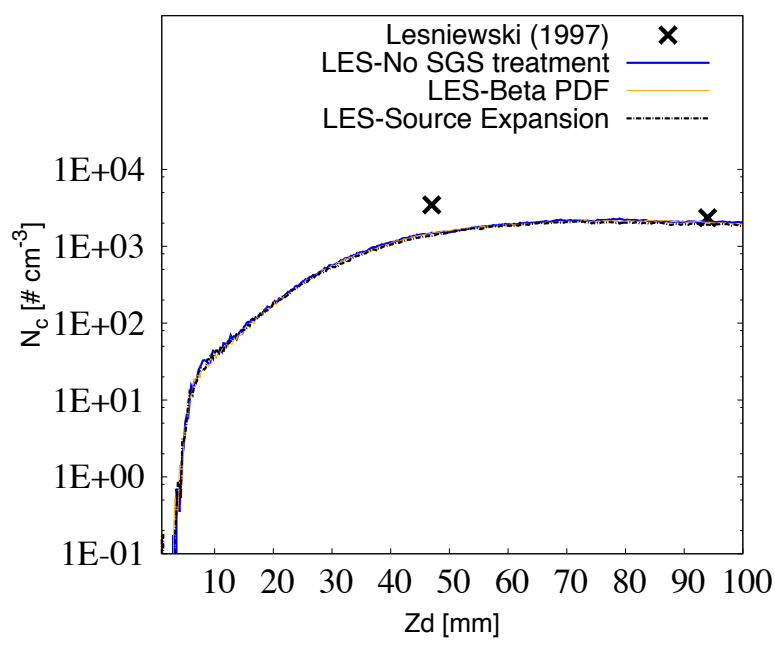

Figure 18: Time average particle concentration on the centreline in the axial direction. The LES results without closure are compared to two different sub grid closure models.

\subsection{Effects of unresolved scales}

The $\beta$-PDF and source expansion methods described in section 3.4.2 were implemented to examine the effects of the sub grid-scales on nucleation rates and particle concentrations. From Fig. 18 it can be seen that there is no significant difference in the particle concentration distributions from the three cases (no model, $\beta$-PDF, and source expansion) in the axial direction along the centreline. However, the cumulative effect in the particle dispersion process may mask a possible discrepancy between models. Therefore a closer examination is required both of nucleation rates and of particle concentrations.

Figure 19 shows the radial distribution of particle concentration at three positions in the domain $\left(Z_{d} / D_{\text {jet }}=5,10\right.$, and 20). Further downstream 


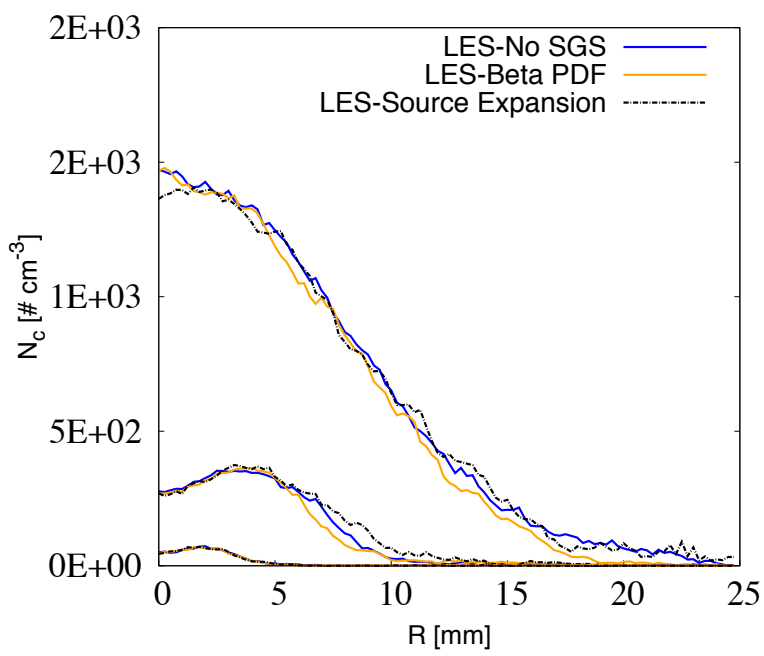

Figure 19: Time average particle concentration in the radial direction at positions $Z_{d} / D_{\text {jet }}=5,10$, and 20 (moving from the bottom left corner to top right).

the particle concentrations are higher, but there seems to be no systematic difference in nucleation rates between the three approaches for the treatment of the SGS fluctuations.

Following a similar analysis to the particle concentrations, Figures 20 and 21 depict the axial and radial LES mean nucleation rates. These values are time-averaged from the instantaneous nucleation rates, as opposed to the RANS equivalent illustrated in Fig. 10 or the EMNM model of [9] which use the mean scalar distribution to calculate the mean nucleation rates. From these figures one may deduce that the differences between the models are small. The inclusion of an SGS closure model results in a slightly higher mean nucleation rate near the peak around $Z_{d}=40 \mathrm{~mm}$ and lower values downstream (figure 20). Similarly, small differences are observed in Fig. 21 


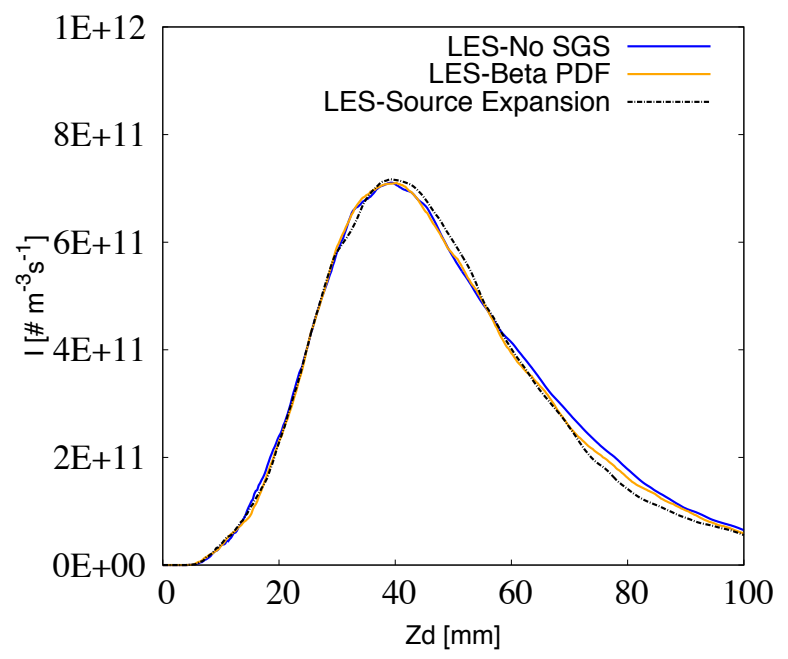

Figure 20: Mean nucleation rate in the axial direction.

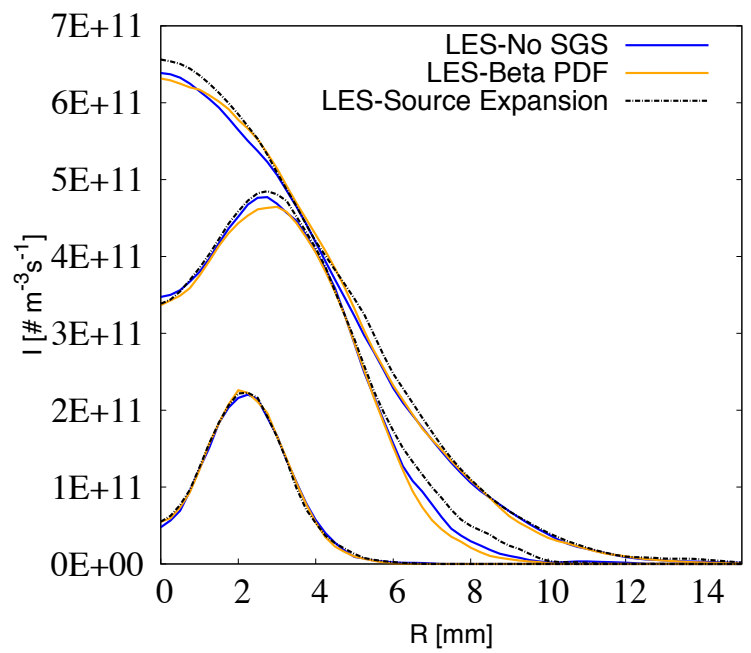

Figure 21: Mean nucleation rate in the radial direction at positions $Z_{d} / D_{j e t}=5,10$, and 20 (moving from the bottom left corner to top right). 

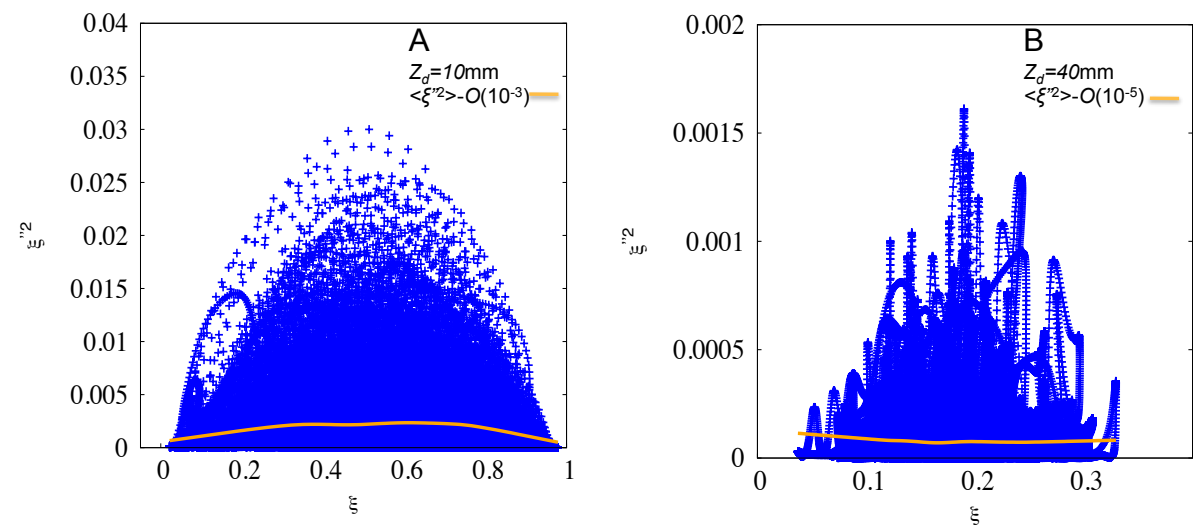

Figure 22: Scatter plot of scalar sub-grid variance versus scalar LES value for the two locations 'A' and 'B' of Fig. $10\left(Z_{d}=10,40 \mathrm{~mm}\right.$, respectively). The solid (yellow) lines represent an ensemble averaged variance $\left\langle\widetilde{\xi^{\prime \prime 2}}\right\rangle_{\xi}$ for every value of $\tilde{\xi}$.

which shows the mean nucleation rates in the radial direction at positions $Z_{d} / D_{j e t}=5,10$, and 20 .

Figure 22 is a scatter plot of the sub grid scalar variance $\widetilde{\xi^{\prime \prime 2}}$ versus the equivalent LES value $\tilde{\xi}$. Data pairs are collected for the two locations of Fig. 10, where $Z_{d}=10$ and $40 \mathrm{~mm}$, respectively. As expected from Fig. 10 the range of values that the scalar $\tilde{\xi}$ acquires in the LES is considerably smaller at location 'B' compared to those at 'A'. For every value of the scalar $\tilde{\xi}$ the equivalent ensemble averaged variance $\left\langle\widetilde{\xi^{\prime \prime 2}}\right\rangle_{\tilde{\xi}}$ is calculated and represented by the solid lines of Fig. 22. The SGS modelled variance is $\widetilde{\xi^{\prime \prime 2}}<<0.1$ in both locations. Therefore (and by comparison to $\dot{I}(\xi)$ in Fig. 8) a negligible variation is observed in the mean nucleation rate because of the modelled sub grid scale fluctuations. A five-fold increase of the model constant $C_{\xi}$ of Eqt. (22) would still result to very small values of the sub grid scalar variance.

From the above results one may draw the following conclusions regarding 
the SGS contribution to nucleation rates. The mean nucleation rate seems to be unaffected by the fluctuations of the scalar within a cell. However, this is not the case for temporal fluctuations: the use of time-averaged scalar values for the calculation of the mean nucleation rate leads to results that are significantly different to the time-averaged instantaneous nucleation rates, as depicted in Fig. 10. Considering the nucleation rate as a source term equivalent to a reaction source term, one may suggest that within a cell of volume $\Delta^{3}$, the sub grid mixing is much faster than the nucleation residence time (i.e. similar to the case of a well-stirred reactor). This can be quantified using an equivalent Damkholer number, $D_{A}^{*}$, defined here as the ratio between an SGS time-scale (conservatively estimated as the integral length scale $l_{I}$ and the RMS velocity) and the nucleation residence time:

$$
D_{A}^{*}=\frac{l_{I} / u_{f, r m s}}{\tau_{n u c}} .
$$

Considering a fluctuation of the order of $10 \%$ of $U_{\text {jet }}$ and the maximum nucleation rate (i.e. the minimum residence time) the time $\tau_{\text {nuc }}$ required for a single particle to form in a cell is of the order of $O\left(10^{-1}\right) \mathrm{s}$, whereas the SGS mixing time-scale is of the order of $O\left(10^{-4}\right)$ s. Therefore $D_{A}^{*}<<1$, suggesting that the LES cell volume is homogeneous and well-stirred with regards to the temperature and DBP concentration. This explains why such small differences are observed when different models are included for the SGS contributions and why temporally-averaged filtered values are unaffected by the unresolved fluctuations. This does not mean that the instantaneous nucleation rates for the three approaches coincide.

Figure 23 shows the instantaneous nucleation rate for three cases, namely: no-SGS, $\beta$-PDF and Source Expansion model. The nucleation rates are eval- 


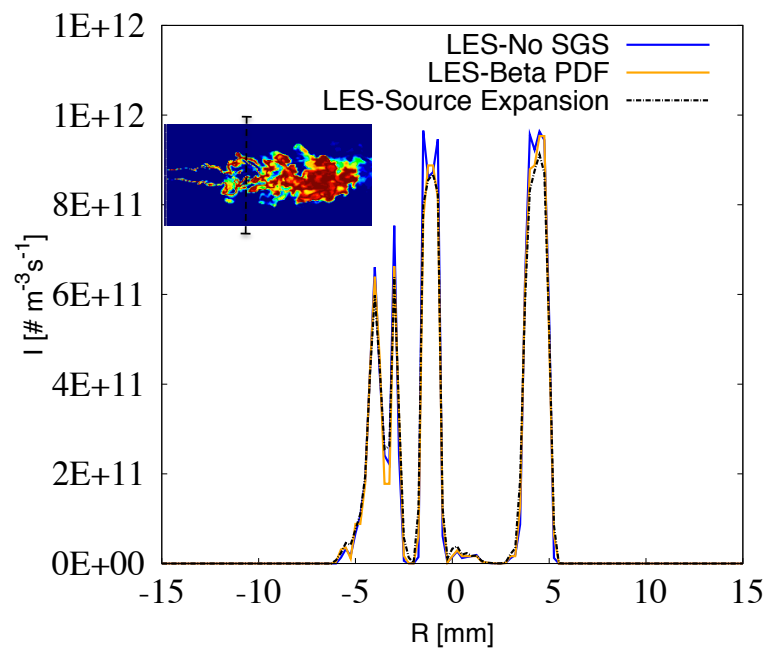

Figure 23: Instantaneous nucleation rate at $Z_{d} / D_{\text {jet }}=10$ calculated from the same LES using the source expansion and $\beta$-PDF methods, and with no model.

uated from the same instantaneous scalar field, at a cross-section of the domain $\left(Z_{d} / D_{\text {jet }}=10\right)$ specified by the dashed line on the contour plot of the same figure. One may notice that the sharp peaks and the sharp changes in the gradient produced by the calculation without a model are smoothened (up to $15 \%$ at $R=-2.5 \mathrm{~mm}$ ) when accounting for the SGS fluctuations.

The two models react similarly, but not identically. The $\beta$-PDF method follows the no-model case closer than the source expansion method. The source expansion method seems to be smoother; this can be attributed to the nature of the model, namely that it performs a Gaussian quadrature filtering.

Arguably, for $L e \neq 1$, the instantaneous filtered nucleation rate would depend on fluctuations of both temperature and DBP concentration, which 
should be treated separately.

Two major conclusions can be extracted for the effects of the SGS contributions on nucleation rates. Firstly, for the particular test case and LES in question the conditions can be considered to be similar to those of a wellstirred reactor. This suggests that the mean filtered values remain unaffected by the filtered-out scales. Secondly, the models do affect the instantaneous results by reducing the peaks and smoothing the sharp gradients.

\section{Conclusions}

As the underlying physical process for many applications, nucleation has received significant attention in both industry and academia. The highly nonlinear nature of the expression for the nucleation rate renders its numerical simulation extremely difficult, but recent increases of computational power have allowed a more in-depth investigation of the phenomenon.

This study has focused on an LES implementation of the nucleation rate provided by the CNT for a turbulent DBP-laden nitrogen jet diffusing in atmospheric air. The main incentives of this work have been to take advantage of the instantaneous rates delivered by the LES and to subsequently evaluate their significance in comparison to the time-averaged values from more traditional approaches. Moreover, the impact of incorporating the effects of unresolved scales due to the spatial filtering have been evaluated using the $\beta$-PDF and source expansion approaches. Having established the numerical treatment and physical characteristics of the test case in question, the results have been presented and discussed.

The proposed parameter-free method for the treatment of the nucleation 
rate as a probabilistic event has been found to predict particle concentrations accurately in comparison to experimental values. The differences between the LES and RANS-equivalent mean nucleation rates have been highlighted, and the advantages of using the former have been discussed. The proposed probabilistic method allows the advantages of LES to be extended to nucleationgoverned parameters, namely particle concentrations.

The problems of measuring nucleation rates experimentally for an open system have been highlighted, thus stressing the importance of computational methods in the study of particle formation. It has been shown that for the particular LES performed here, the effects of the unresolved scales are insignificant for time-averaged mean values. The $\beta$-PDF and source expansion methods predicted very similar particle concentrations and nucleation rates in the radial and axial directions. Considering the particle nucleation rate as a source term equivalent to a chemical reaction source term, an order-ofmagnitude analysis of the time-scales involved has shown that the case could be considered to be well-stirred within the volume of a cell; this may explain the minimal effect of the sub grid-scales on the mean LES values. However, by closer examination of the instantaneous values it has been shown that the models do affect the results.

Both sub grid-scale models reduce peaks and smooth gradients when compared to the instantaneous nucleation rate calculated without a model, where the source expansion method appears to have a more pronounced effect. No significant difference could be seen for the particle concentrations. This becomes important when comparing to alternative approaches for the simulation of nucleation rates, where it has been shown that the exclusion of fluc- 
tuations (temporal in RANS and spatial in the EMNM) leads to significant differences in the calculation of particle nucleation rate and concentration.

\section{References}

[1] S. Peoples, C. Thurn, J. Steinmetz, W. Oliver, Assessment of fugitive emissions of proc from petroleum refining operations : final report, Tech. rep., Sacramento, Calif: Radian Corp (1987).

[2] S. E. Pratsinis, W. Zhu, S. Vemury, The role of gas mixing in flame synthesis of titania powders, Powder Technology 86 (1996) 87-93.

[3] Y. Sung, V. Raman, R. Fox, Les based multiscale modelling of tio2 nanoparticle synthesis in a turbulent flame reactor using detailed nucleation chemistry, Chemical Engineering Science 66(19) (2011) 4370-4381.

[4] G. Y. D. Veroli, S. Rigopoulos, Modeling of aerosol formation in a turbulent jet with the transported population balance equation-probability density function approach, Phys. Fluids 23 (2011) 043305-1.

[5] W. Koch, T. Wind, N. Karfich, Modeling and experimental evaluation of an aerosol generator for very high number currents based on a free turbulent jet, J. Aerosol Sci 24 (1993) 909-918.

[6] J. Lighty, J. Veranth, A. Sarofim, Combustion aerosols: Factors governing their size and composition and implications to human health, Journal of the Air and Waste Management Association 50 (2000) 1565-1618.

[7] P. Reist, Introduction to aerosol science, Macmillan Pub Co., 1984. 
[8] A. Laaksonen, V. Talanquer, D. Oxtoby, Nucleation: Measurements, theory, and atmospheric applications, Annu. Rev. Phys. Chem. 46 (1995) $489-524$.

[9] K. Zhou, T. Chan, Simulation of homogeneous particle nucleation in a free turbulent jet, Aerosol Science and Technology 45:8 (2011) 973-987.

[10] T. Lesniewski, S. Friedlander, Particle nucleation and growth in a free turbulent jet, Proc. R. Soc. London Ser. A 454 (1998) 2477-2504.

[11] J. Brock, P. Kuhn, Condensation aerosol formation and growth in a laminar coaxial jet, J. Aerosol Sci 17 (1986) 11-22.

[12] K. Okuyama, Y. Kousaka, D. Warren, R. Flagan, J. Seinfeld, Homogeneous nucleation by continuous mixing of high temperature vapor with room temperature gas, Aerosol Science and Technology 6 (1987) 15-27.

[13] M. Anisimov, K. Hameri, M. Kulmala, Construction and test of laminar flow diffusion chamber: Homogeneous nucleation of dbp and n-hexanol, J. Aerosol Sci 25:1 (1994) 23-32.

[14] K. Hameri, M. Kulmala, E. Krissinel, G. Kodenyov, Homogeneous nucleation in a laminar flow diffusion chamber: The operation principles and posibilities for quantitative rate measurements, J. Chem. Phys. 105 (1996) 7683.

[15] M. Wilck, K. Hameri, F. Stratmann, M. Kulmala, Determination of homogeneous nucleation rates from laminar-flow diffusion chamber data, J. Aerosol Sci 29:8 (1998) 899-911. 
[16] V. Mikheev, N. Laulainen, S. Barlow, The laminar flow tube reactor as a quantitative tool for nucleation studies: Experimental results and theoretical analysis of homogeneous nucleation of dibutylphthalate, J. Chem. Phys. 113 (2000) 3704.

[17] R. So, B. Hwang, On similarity solutions for turbulent and heated round jets, Journal of Applied Mathematics and Physics 37 (1986) 624.

[18] H. Hussein, S. Capp, W. George, Velocity measurements in a highreynolds-number, momentum-conserving, axisymmetric turbulent jet, Journal of Fluid Mechanics 258 (1994) 31-75.

[19] P. Chu, J. Lee, V. Chu, Spreading of turbulent round jet in coflow, J. Hydraul. Eng. 125 (1999) 193-204.

[20] T. Lesniewski, S. Friedlander, The effect of turbulence on rates of particle formation by homogeneous nucleation, Aerosol Science and Technology 23 (1995) 174-182.

[21] T. Lesniewski, Particle nucleation and growth in turbulent jets, Ph.D. thesis, University of California (1997).

[22] A. Garmony, E. Mastorakos, Aerosol nucleation and growth in a turbulent jet using the stochastic fields method, Chemical Engineering Science 63 (2008) 4078-4089.

[23] A. Kempf, B. Geurts, J. Ofelein, Error analysis of large-eddy simulation of the turbulent non-premixed sydney bluff-body flame, Combust. Flame 158 (2011) 2408-2419. 
[24] M. Pettit, B. Coriton, A. Gomez, A. Kempf, Large eddy simulation and experiments on non-premixed highly turbulent opposed jet flows, Proc. Combust. Inst 33 (2011) 1391-1399.

[25] N. Murfield, S. Garrick, The effects of unresolved scalar fluctuations during homogeneous nucleation, Aerosol Science and Technology 47(7) (2013) 806-817.

[26] J. Smagorinsky, General circulation experiments with the primitive equations, i. the basic experiment., Monthly Weather Review 152 (1963) 9199.

[27] D. Lilly, The representation of small-scale turbulence in numerical simulation experiments, Proc. of the IBM Scientific Computing Symp. on Environmental Sciences 1 (1967) 195-210.

[28] R. Courant, K. Friedrichs, H. Lewy, On the partial difference equations of mathematical physics, Mathematische Annalen 100 (1928) 32-74.

[29] A. Kempf, M. Klein, J. Janicka, Efficient generation of initial and inflow conditions for transient turbulent flows in arbitrary geometries, Flow Turbulence and Combust 74 (2005) 67-84.

[30] M. Klein, A. Sadiki, J. Janicka, A digital filter based generation of inflow data for spatially developing direct numerical or large eddy simulations, Journal of Computational Physics 186 (2003) 652-665.

[31] W. Jones, D. Sheen, A probability density function method for modelling liquid fuel sprays, Flow Turbulence and Combustion 63 (1999) 379-394. 
[32] M. Bini, W. P. Jones, Large eddy simulation of particle laden turbulent flows, J Fluid Mech 614 (2008) 207-252.

[33] W. Jones, S. Lyra, S. Navarro-Martinez, Large eddy simulation of a swirl stabilized spray flame, Proc. Combust. Inst. 33 (2011) 2153-2160.

[34] I. Pesmazoglou, A. Kempf, S. Navarro-Martinez, A dynamic model for the lagrangian stochastic dispersion coefficient, Phys. Fluids 25 (2013) 125108 .

[35] M. Bini, W. P. Jones, Particle acceleration in turbulent flows: A class of nonlinear stochastic models for intermittency, Phys. Fluids 19 (2007) 035104-1.

[36] S. Lyra, Large eddy simulation of isothermal and reacting sprays, Ph.D. thesis, Imperial College London (2010).

[37] L. Zaichik, V. Alipchenkov, A. Avetissian, A statistical model for predicting the heat transfer of solid particles in turbulent flows, Flow Turbulence Combust 86 (2011) 497-518.

[38] M. C. Yuen, L. W. Chen, On drag of evaporating liquid droplets, Comb. Sci. \& Tech. 21 (1976) 537-542.

[39] R. Becker, W. Doring, Kinetische behandlung der keimbildung in ubersattingten damfen, Ann. Phys. 24 (1935) 719-52.

[40] J. Frenkel, A general theory of heterophase fluctuations and pretransition phenomena, J. Chem. Phys. 7 (1939) 538. 
[41] J. Zeldovich, Theory of the formation of a new phase, J. Expl. Theoret. Physics (USSR) 12 (1942) 525.

[42] J. Schmelzer (Ed.), Nucleation theory and applications, Wiley-VCH, 2005.

[43] M. Jacobson, Fundamentals of atmospheric modeling, Cambridge University Press, 1999.

[44] AIChE, Thermodynamical database, Tech. rep., American Institute of Chemical Engineers: Design Institute for Physical Property Data (1997).

[45] V. Bedanov, V. Vaganov, G. Gadiyak, G. Kodenev, E. Rubakhin, The experimental determination of number of molecules in the critical embryo, Khimicheskaya Fisika 7 (1988) 555-563.

[46] M. Potin-Gautier, P. Grenier, J. Bonastre, Nouvelle application analytique de la methode de determination des pressions de vapeur par saturation dun gaz inerte, Anal. Lett. 15 (1982) 1431.

[47] J. Lothe, G. Pound, Reconsiderations of nucleation theory, J. Chem. Phys. 36 (1962) 2080-85.

[48] H. Reiss, Treatment of droplike clusters by means of the classical phase integral in nucleation theory, J. Stat. Physics 2 (1970) 83-104.

[49] S. Girshick, C. Chiu, Kinetic nucleation theory: A new expression for the rate of homogeneous nucleation from an ideal supersaturated vapor, J. Chem. Phys. 93 (1990) 1273. 
[50] A. Dillman, G. Meier, A refined droplet approach to the problem of homogeneous nucleation from the vapour phase, J. Chem. Phys. 94 (1991) 3872-3884.

[51] A. Laaksonen, I. Ford, M. Kulmala, Revised parametrisation of the dillman-meier theory of homogeneous nucleation, Phys. Rev. E 49 (1994) $5517-5524$.

[52] B. Hale, Application of a scaled homogeneous nucleation rate formalism to experimental data at T $<<$ Tc, Phys. Rev. A 33 (1986) 4156.

[53] L. Granasy, Diffuse interface theory of nucleation, Journal of NonCrystalline Solids 162:3 (1993) 301-303.

[54] J. Wu, S. Menon, Aerosol dynamics in the near field of engine exhaust plumes., Journal of Applied Metereology 40 (2001) 795-809.

[55] S. Rigopoulos, A. Jones, Finite-element scheme for solution of the dynamic population balance equation, AICHE J 49 (2003) 1127-1139.

[56] S. Pratsinis, Simultaneous nucleation, condensation and coagulation in aerosol reactors, Journal of Colloid and Interface Science 124(2) (1988) $416-427$.

[57] S. Rigopoulos, Pdf method for population balance in turbulent reactive flow, Chemical Engineering Science 62 (2007) 68656878.

[58] G. D. Verolli, S. Rigopoulos, Modeling of turbulent precipitation: A transported population balance-pdf method, AICHhE J. 56 (2010) 878. 
[59] J. McDonald, Homogeneous nucleation of vapor condensation. i. thermodynamic aspects, Americal Journal of Physics 30 (1962) 870.

[60] H. Risken, The Fokker Planck Equation, Springer, 1984.

[61] J. Wedekind, R. Strey, D. Reguera, New method to analyze simulations of activated processes, J. Chem. Phys. 126 (2007) 134103.

[62] A. Fager, J. Liu, S. Garrick, Hybrid simulations of metal particle nucleation: A priori and a posteriori analyses of the effects of unresolved scalar interactions on nanoparticle nucleation, Phys. FLuids 24 (2012) 075110.

[63] R. Fox, On the relationship between lagrangian micromixing models and computational fluid dynamics, Chemical Engineering and Processing 37 (1998) 521-535.

[64] D. Pope, Pdf methods for turbulent reactive flows, Progress in Energy and Combustion Science 11 (1985) 119-192.

[65] J. Minier, E. Peirano, The pdf approach to turbulent polydispersed twophase flows, Physics Reports 352 (2001) 1-214.

[66] D. Haworth, Progress in probability density function methods for turbulent reacting flows, Progress in Energy and Combustion Science 36 (2010) 168-259.

[67] J. Floyd, A. Kempf, A. Kronenburg, R. Ram, A simple model for the filtered density function for a passive scalar in combustion les, Combust. Theor. Modeling 13(4) (2009) 559-588. 
[68] D. Marchisio, R. Fox, A. Baressi, G. Baldi, On the comparison between presumed and full pdf methods for turbulent precipitation., Ind. Eng. Chem. Res. 40 (2001) 5132-5139.

[69] J. Baldyga, W. Orciuch, Closure method for precipitation in inhomogeneous trurbulence, Proc. 14th Symposium on Industrial Crystallization 86 (1999) 1069.

[70] H. Feng, M. Olsen, R. Fox, J. Hill, Conditional statistics for passivescalar mixing in confined rectangular jet, Phys. Fluids 19 (2007) 055104.

[71] S. Girimaji, Assumed beta-pdf model for turbulent mixing: Validation and extension to multiple scalar mixing, Comb. Sci. \& Tech. 78 (1991) 177.

[72] H. Feng, M. Olsen, R. Fox, J. Hill, Conditional statistics of passive-scalar mixing in confined wake flow, Phys. Fluids 20 (2008) 077105-1.

[73] H. Wang, K. Luo, J. Fan, Direct numerical simulation and cmc (conditional moment closure) sub-model validation of spray combustion, Energy 46 (2012) 606-617.

[74] R. Baurle, S. Girimaji, Assumed pdf turbulence-chemistry closure with temperature-composition correlations, Combust. Flame 134 (2003) 131148.

[75] M. Abramowitz, I. Stegun (Eds.), Handbook of Mathematical Functions: with Formulas, Graphs, and Mathematical Tables, U.S. Department of Commerce, National Bureau of Standards, 1964. 
[76] C. Pierce, P. Moin, A dynamic model for sub-grid scale variance and dissipation rate of a conserved scalar, Phys. Fluids 10:12 (1998) 30413044 .

[77] N. Branley, W. Jones, Les of turbulent non-premixed flame, Combust. Flame 127 (2001) 1914-1934.

[78] R. Kulkarni, W. Polifke, Large eddy simulation of autoignition in a turbulent hydrogen jet flame using a progress variable approach, Journal of Combustion 2012 (2012) 11.

[79] K. Vogiatzaki, S. Navarro-Martinez, Direct modelling of the filtered source term by series expansion, in: 13th International Conference on Numerical Combustion, Crete, Greece, 2011.

[80] P. Sagaut, Large Eddy Simulation for incompressible flows, Springer, 1998.

[81] M. Germano, U.Piomelli, M. P., W. Cabot, A dynamic sub-grid-scale eddy viscosity models, Phys. Fluids 23 (1986) 1760-1765.

[82] D. Dowling, P. Dimotakis, Similarity of the concentration field of gasphase turbulent jets, J. Fluid Mech. 218 (1990) 109-141. 